\begin{tabular}{|c|l|}
\hline Title & A measuring system for the time variation of size and charge of a single spherical particle and its applications \\
\hline Author(s) & Nakajima, Y oji \\
\hline Citation & $\begin{array}{l}\text { Chemical Engineering Science, 61/7), 2212-2229 } \\
\text { https://doi.org/10.1016/.ces.2005.05.004 }\end{array}$ \\
\hline Issue Date & 2006-04 \\
\hline Doc URL & http://hdl.handle.net/2115/5846 \\
\hline Type & article (author version) \\
\hline File Information & CES61-7.pdf \\
\hline
\end{tabular}

Instructions for use 


\title{
A measuring system for the time variation of size and charge of a single spherical particle and its applications
}

\author{
Yoji NAKAJIMA \\ Division of Materials Science and Engineering, Graduate School of Engineering, \\ Hokkaido University, Sapporo, 060, Japan. \\ E-mail:yoji@eng.hokudai.ac.jpＦax:+81-(0)11-706-6593
}

\begin{abstract}
A single charged particle is trapped in a simple quadrupole electrode assembly and oscillated by means of a controlled electric field. A laser Doppler velocimeter (LDV) of the fringe mode is
\end{abstract} used for detecting the amplitude and phase lag of the particle oscillation with respect to the driving ac field. A unique method for the LDV signal processing is presented that takes the advantage of the sinusoidal oscillation of the particle at a known frequency. Superimposed to the ac drive, a dc drive is added for highly accurate measurements of particle size and charge. This enables us also to discriminate the polarity of charge without Bragg cells.

In this paper, the basic principle of the method of the size and the charge measurements is explained and the accuracy of the measurements is demonstrated experimentally. The errors in the size and charge measurements, respectively, are less than $1 \%$ and $3 \%$ with a confidence coefficient of $99 \%$. Since this apparatus repeats the measurements every 0.3 second for a single particle, the errors can be reduced to $0.1 \%$ and $0.3 \%$ when the measured values over a period of 30 seconds, or over 100 data are averaged.

As some areas of its applications, experimental data are presented on the Rayleigh instability of evaporating charged droplets. It is shown that there are three types of Rayleigh 
fission. One of the types seemed to show occurrence of air breakdown around a micron sized spherical particle, which has not been reported so far. However experiments in highly insulating gas $\left(\mathrm{SF}_{6}\right)$ revealed that it was not the case but a type of Rayleigh fission. Nevertheless the experimental results gave important information on the charge limit of spherical solid particle due to electric breakdown in air at normal room conditions.

Some cares to ensure the advantages of the present method are presented, and possible improvements of the apparatus are also suggested for future use.

Author keywords : charged droplet, charged particle, charge measurement, size measurement, LDV, electrodynamic trapping, Rayleigh fission 


\section{Introduction}

Measurement of time variations in particle size and charge properties often yields novel information. For instance, evaporation characteristics of small droplets (Taflin et al., 1988, Shulman et al., 1997, Widman et al., 1998), uptake dynamics of $\mathrm{HCl}$ by a single sulfuric acid microdroplet (Schwell et al., 2000), the Rayleigh instability of charged droplets (Duft et al., 2002, Taflin et al., 1989), chemical reaction rate constant of droplets (Ward et al., 1989) have been studied from the measurement of the time variation in size of a single droplet. For these purposes, the electrodynamic balances (EDB) have been used to trap and to levitate a single aerosol particle freely in a gaseous medium. In the experiments cited above, bihyperboloidal electrodes (Wuerker et al., 1959) with complex design and construction were used, so that the electric field formed by the electrodes could be readily calculable. However, such bihyperboloidal electrodes are rather costly to construct, and moreover, the electrode configuration restricts the scattered light collection angle for the optical receiver within a rather narrow limit.

Instead, simpler forms of electrodes have been accepted for EDBs. Richardson et al. (1989) used a cylindrical and two hemispherical cap electrodes to measure the Rayleigh instability of charged droplets. Davis and Bridges (1994) examined the cause of charged droplet fission prior to reaching the Rayleigh instability limit by using two different sets of double ring electrodes. Bhanti and Ray (1998) used a similar type EDB to Richardson's for the measurement of instantaneous chemical reaction rate constant in a droplet. Zhu et al. (2002) measured mass transfer from an oscillating small droplet using an octopole double ring electrode configuration. 
In the present system, a more facile electrode configuration, namely two parallel rod electrodes located in the central plane of a pair of parallel plate electrodes, is adopted for forming a quasi-quadrupole ac electric field, which can trap charged particles at the central portion of the electric field. The parallel plate electrodes also provide a uniform ac electric field to oscillate the charged particle.

In the experiments cited above, the droplet size was measured by using two elaborate optical methods based on the Mie light scattering theory. One is now called the phase function method (Bartholdi et al., 1980) and the other is called the optical resonance method (Ashkin and Dziedzic, 1981). Both the methods, in particular the latter, offer very accurate size measurement if the refractive index of the droplet is known precisely. But both the methods require very complicated and time-consuming computations. For several decades, hydrodynamic approaches for measuring the sizes of cloud droplets and smoke particles have been developed. Wells and Gerke (1919) utilized photographs of particle oscillation in an ac field for the first time, though they had to assume the charge of the droplet. Using a laser Doppler velocimeter, Mazumder and Kirsch (1977) and Renninger et al. (1981) proposed a novel method, in which the phase lag of the motion of particle relative to the oscillating driving force was related to the relaxation time or aerodynamic size of the particle. This system is now known as the E-SPART (electrical single particle aerodynamic relaxation time) analyzer.

In the present measuring system, a quasi-quadrupole electrode system mentioned above is incorporated in the measurement volume of a laser Doppler velocimeter of the fringe mode. The 
basic idea of the system is similar to that of the E-SPART analyzer, but the equipment is extremely simplified; it uses neither conventional LDV signal demodulator nor Bragg cells. Nevertheless the polarity of droplet charge can be discriminated if necessary, and further, the accuracy of the size and charge measurements is comparable to that attained by EDBs owing to the special method of LDV signal analysis and also to the repeated measurement for a single particle. In this paper, the unique features in the basic principle of the measuring system are elucidated and accuracy of the size and charge measurements is demonstrated.

This measuring system has been developed for the experiment of droplet agglomeration enhanced by electrostatic force and particle vibration (Nakajima and Sato, 2003). To exemplify other applications, some experimental results on the Rayleigh instability are presented, which show the complexity of the Rayleigh disruption of charged droplets. An attempt to clarify the charge limit for a spherical solid particle sustainable in normal air follows. Then a semi-empirical equation relating the particle diameter and the charge limit for negatively charged particles is proposed on the basis of Townsend theory. Although the theory of Townsend is not applicable to positively charged particles, an experiment in argon gas showed that the difference due to the polarity was not very significant, and the prediction may be usable for both positively and negatively charged particles.

\section{Theoretical descriptions of the measuring system}

\subsection{Trapping electrodes}


Wuerker et al. (1959) analyzed and examined the stability of charged particle motion in an exact bihyperboloidal electrode configuration to clarify the basis of electrodynamic trapping of charged particles. Here the simplicity of the trapping system is demonstrated because we might presume that a very sophisticated electrode configuration would be necessary for the trapping system.

In this experiment, a simplified electrode configuration as shown in Fig.1 was used to trap and to levitate a charged particle at the center of the measurement volume of LDV. It consisted of a pair of parallel plates and two rods with two insulated spherical electrodes for each rod. An ac voltage was applied to the rods to form a quasi-quadrupole ac electric field, which electrodynamically trapped charged particles as in the quadrupole mass spectrum analyzers. The plate electrodes behaved like mirrors making the electric images of the rod electrodes. For analyzing the electric field distribution, the electric image method modified by the charge simulation method was used. Fig.2 shows the equipotential and electric force lines thus obtained. As shown at the center of Fig.2, a saddle point in the $\mathrm{x}-\mathrm{z}$ plane is formed along the centerline of the electrode system. The electric field around the saddle point is described below.

The absolute value (or norm) of the electric field vector in the area enclosed by the dotted square in Fig.2 is illustrated in Fig.3. The square $(6 \times 6 \mathrm{~mm})$ covers enough wide area in comparison with the diameter of the measurement volume, which was approximately $2 \mathrm{~mm}$ in diameter. As is seen from Fig.3, the absolute value forms a cone. It follows that the components of the electric field can be approximated by: 


$$
E_{x} \approx A_{x} x \sin \omega t, \quad E_{z} \approx A_{z} z \sin \omega t
$$

where $x$ and $z$ are the horizontal and the vertical coordinates as shown in Fig.2. The angular frequency of the ac voltage applied to the rod electrodes is denoted by $\omega$, and $A_{\mathrm{x}}$ and $A_{\mathrm{z}}$ are the proportionality constants. In Eq.(1) $A_{z}$ should be $-A_{x}$ because the electric field must satisfy the Laplace equation.

The position of a charged particle, $(x, z)$, in this field may be written as follows, provided that the particle is so small that we can presume a linear relationship between the drag force and particle velocity:

$$
m \frac{\mathrm{d}^{2} x}{\mathrm{~d} t^{2}}+R \frac{\mathrm{d} x}{\mathrm{~d} t}=q E_{x}, \quad m \frac{\mathrm{d}^{2} z}{\mathrm{~d} t^{2}}+R \frac{\mathrm{d} z}{\mathrm{~d} t}=q E_{z}-m g
$$

where $m$ is the particle mass, $R$ the drag coefficient, $q$ the particle charge, and $g$ the acceleration constant due to gravity. Here we notice from Eq.(1) that $E_{\mathrm{x}}$ does not depend on $z$, and $E_{\mathrm{z}}$ is independent of $x$. There are various structures of electrodes that can form such an electric field although valid only locally, where every component of the electric field is nearly proportional to the corresponding coordinate centered at the saddle point. Thus the equations can be solved for $x$ and $z$ independently.

In case the charged particle oscillates around $z_{0}$ with a small amplitude, we may put $E_{z} \approx A_{z} z_{0} \sin (\omega t)$ in Eq.(2). Then we have an approximate solution for $z$,

$$
z=-\frac{q A_{z} z_{0}(m \omega \sin \omega t+R \cos \omega t)}{\omega\left(R^{2}+m^{2} \omega^{2}\right)}+z_{0}
$$


Hence the driving force in $z$-direction becomes

$$
\begin{gathered}
q A_{\mathrm{z}} z \sin (\omega t)-m g=-\frac{q^{2} A_{\mathrm{z}}^{2} m z_{0}}{2\left(R^{2}+m^{2} \omega^{2}\right)}-m g+q A_{\mathrm{z}} z_{0} \sin (\omega t)+\frac{q^{2} A_{\mathrm{z}}^{2} z_{0} m}{2\left(R^{2}+m^{2} \omega^{2}\right)} \cos (2 \omega t) \\
-\frac{q^{2} A_{\mathrm{z}}^{2} R z_{0}}{2 \omega\left(R^{2}+m^{2} \omega^{2}\right)} \sin (2 \omega t)
\end{gathered}
$$

The first term in the right hand side of Eq.(4) is a steady force yielded by the electrodynamic effect. This term is positive when $z_{0}<0$ and gives the charged particle a levitation force. At the equilibrium state, it balances with the gravitational force, $m g$. As for $x$-direction, the same calculation shows that the electrodynamic effect exerts a steady force directing to $x=0$ on the charged particle. In this manner, charged particles are trapped near the centerline of the two parallel rod electrodes.

As shown in Fig.1, a bias dc voltage on the ac trapping voltage is applied to the spherical electrodes so that the charged particles drift along the centerline (i.e., $y$-axis) towards the center of the electrodes by electrostatic repulsion. Another dc bias applied to the parallel plate electrodes may help dislocated particles to turn back to the trapping zone. The polarity of the dc biases in Fig.1 is for positively charged particles, and all the Zenner diodes should be reversed in polarity for negatively charged particles.

\subsection{Amplitude and phase measurement by LDV}

As is well known, the phase lag of the oscillation of a particle driven by an acoustic or ac electric field reflects the size of the particle. If we use an ac electric field, the amplitude of the particle 
oscillation gives information on the charge of the particle as well. Fig.4 is an example of the LDV light signal (beat signal) scattered from a spherical particle oscillating in the interference fringes formed by two coherent laser beams. The sinusoidal curve in the figure refers to the driving ac voltage for particle oscillation.

In this example, the amplitude, $A$, is several times larger than the fringe spacing, $\delta$, and so we can readily notice in the beat signal the points at which the particle motion reverses. Hence the phase lag of particle displacement, $\phi$, with respect to the driving ac voltage can be determined as depicted in the figure. Further, by counting the number of peaks between the reversing points and using a suitable interpolation method, we can estimate the amplitude of the particle oscillation. This method is straightforward but adequate only for amplitude greater than the fringe spacing (Sato and Nakajima, 1990), and it is not necessarily suitable for the present measurement.

As the hydrodynamic formula relating the phase lag to the particle size is appropriate only for small amplitude of the particle oscillation, the amplitude should be as small as possible for precise measurement. When the amplitude becomes small enough for the precise measurement, the waveform of the beat signal looks like a sinusoidal wave as shown in Fig.5. To extract adequate information for the phase and amplitude measurements from the beat signal, we aim to expand the signal in a Fourier series as below.

As will be explained later, a superposition of a suitable dc bias on the ac electric field for driving the charged particle is advantageous for the present method. (The dc bias circuit is not 
shown in Fig.1.) Then the location, $x$, of the particle in the measurement volume of the LDV is supposed here to be

$$
x=A \sin \left(\omega_{\mathrm{p}} t-\phi\right)+u t+x_{0}
$$

where $A$ is the displacement amplitude, $\omega_{\mathrm{p}}$ the angular frequency of particle oscillation, $\phi$ the phase lag of particle displacement with respect to the driving ac voltage, $u$ the steady velocity, $t$ the time, and $x_{0}$ the initial location of the particle. Provided that the diameter of the measurement volume is much larger than the displacement of the moving particle, the light intensity across the fringe planes may be assumed to vary in a cosine wave. Hence the beat signal, namely the scattered light intensity from the moving particle in the fringes, may be written as

$$
\mathrm{i}_{p}=B \cos (k x)=B \cos \left\{k\left(u t+x_{0}\right)\right\} \cos \left\{k A \sin \left(\omega_{\mathrm{p}} t-\phi\right)\right\}-B \sin \left\{k\left(u t+x_{0}\right)\right\} \sin \left\{k A \sin \left(\omega_{\mathrm{p}} t-\phi\right)\right\}
$$

where $B$ is the constant that depends mainly on the optical system and the particle size. The constant $k$ is the wave number of the fringes, i.e., $2 \pi$ divided by the fringe spacing $\delta$. In reality, a very low frequency (or even dc) component termed the pedestal is accompanied. More precise explanation of the beat signal including the pedestal for the fringe mode LDV may be found elsewhere (Roberds, 1977), but Eq.(6) is practical enough because the pedestal component in beat signal is not used in the present method.

To expand Eq.(6) in a Fourier series, use is made of the following series expansions (Pipes 
and Harvill, 1970) i.e.,

$$
\cos \{a \sin (b)\}=J_{0}(a)+2 \sum_{n=1}^{\infty} J_{2 n}(a) \cos (2 n b), \quad \sin \{a \sin (b)\}=2 \sum_{n=1}^{\infty} \mathrm{J}_{2 n-1}(a) \sin \{(2 n-1) b\}
$$

where $J_{\mathrm{n}}(a)$ is the Bessel function of the first kind and of order $n$. Then we have

$$
\begin{aligned}
& \mathrm{i}_{p}=B J_{0}(k A) \cos \left(k u t+k x_{0}\right) \\
& +B \sum_{n=1}^{\infty} J_{2 n-1}(k A)\left[\cos \left\{(2 n-1) \omega_{\mathrm{p}} t+k u t-(2 n-1) \phi+k x_{0}\right\}-\cos \left\{(2 n-1) \omega_{\mathrm{p}} t-k u t-(2 n-1) \phi-k x_{0}\right\}\right] \\
& +B \sum_{n=1}^{\infty} J_{2 n}(k A)\left[\cos \left\{2 n \omega_{\mathrm{p}} t+k u t-2 n \phi+k x_{0}\right\}+\cos \left\{2 n \omega_{\mathrm{p}} t-k u t-2 n \phi-k x_{0}\right\}\right]
\end{aligned}
$$

This shows that the spectrum of the beat signal consists of infinite number of spectral lines located at the angular frequencies of $k u, \omega_{\mathrm{p}} \pm k u, 2 \omega_{\mathrm{p}} \pm k u, 3 \omega_{\mathrm{p}} \pm k u$, and so on. The intensity of each line is proportional to $J_{\mathrm{n}}(k A)$, and hence the amplitude, $A$, determines the spectral distribution.

To elucidate the way for calculating the amplitude and the phase lag of the particle oscillation from the beat signal, the data shown in Fig.5 is processed as an example. The abscissa of the figure is the time. The sampling rate was $(1 / 15) \mu \mathrm{s}^{-1}$. Although the data was truncated at 1000 (=15ms) in the figure, the actual record consisted of 5000 data $(=75 \mathrm{~ms})$, which included about 85 cycles of the particle oscillation. The frequency of the particle oscillation was $1131 \mathrm{~Hz}$. During the beat signal was transmitted to a computer for recording, an automatic control system intermitted the trapping ac voltage and superimposed a suitable dc component on the driving ac voltage so as to make $k u / 2 \pi \approx 1131 / 4$. This resulted in an equally spaced spectrum lines as may 
be seen from Eq.(8). As the measurement was carried out for a single particle repeatedly, such a control was possible by referring to the preceding measurement. Further explanations of the automatic control system are omitted here.

The spectrum was calculated by using DFT (discrete Fourier transformation). Before the DFT analysis, the hanning window (Blackman and Tuckey, 1958) was applied to the raw data to avoid the unfavorable effect of limited length of data and noises. The result is shown in Fig.6. We find spectrum lines at frequencies of $291 \mathrm{~Hz}, 840 \mathrm{~Hz}, 1423 \mathrm{~Hz}, 1972 \mathrm{~Hz}, 2555 \mathrm{~Hz}$, and so on. These conform very well to the frequencies predicted by Eq.(8) if we take $k u / 2 \pi$ to be $291 \mathrm{~Hz}$. Eq.(8) implies that the intensity of each line spectrum should be proportional to $J_{\mathrm{n}}(k A)$, where $n$ is the ordinal number of the harmonics corresponding to the frequencies. Except for $n=0$, each harmonic should have two frequency components (spectrum lines) with the same intensity. Fig.6 also reflects this fact. As $k A$ determines the intensity distribution of the spectrum, we can find the amplitude of the particle oscillation, $A$, from the spectrum. To do this, the measured intensity of spectrum line for the n-th harmonic was divided by the sum of seven intensities for harmonics from 0th to 6th to yield the normalized intensity:

$$
h(n)=\left|J_{n}(k A)\right| / \sum_{m=0}^{6}\left|J_{m}(k A)\right|
$$

Fig.7 shows the theoretical dependence of the intensities on $k A$, though it includes only harmonics up to 4th to avoid graphical complexity. Since the higher order components die out for small $k A$, the intensity of 0 th order plays important role. If the steady component hadn't been 
superimposed on the oscillating particle motion, the two frequency components in each harmonic order would degenerate into a single component. As a result, the 0th order component in Fig.6 would have been submerged in the pedestal component. For this reason the superposition of a suitable dc component on the ac driving voltage is preferable for the measurement of small amplitude.

Now let's seek for the value of $k A$ that fits the spectrum distribution in Fig.6. We see that the second harmonic has the highest intensity, the 0th and the 3rd come after it with almost the same intensity, the 4th follows them and the 1st is the lowest among them. This situation can be found in Fig.7 at $k A=2 \pi A / \delta=3.5$ as indicated by the broken vertical line. The fringe spacing, $\delta$, was $9.5 \mu \mathrm{m}$ in this measurement and so the amplitude was evaluated to be $5.29 \mu \mathrm{m}$. In the actual system, the theoretical intensity distributions $h(n)$ in Eq.(9) for $n$ from 0 to 6 were tabulated in a computer for various $k A$, and the observed intensity distribution was compared with them to find the value of $k A$ that gave the best-fit distribution by using the least mean square technique. This method was practical for the amplitude range $0.1 \leq k A \leq 20$.

The phase measurement is straightforward. As is readily seen from Eq.(8), we have two frequency components for the first order harmonic, of which phase lags are $\phi+k u$ and $\phi-k u$. We can calculate them from the results of DFT for corresponding frequencies to give $2 \phi$ by addition. The use of hanning window turned out very effective for precise phase measurement. In case $\left|J_{1}(k A)\right|<\left|J_{2}(k A)\right|$, it is preferable to use the second harmonic for the phase measurement.

In practice, however, the value of $\phi$ varies between $\pi / 2$ and $\pi$ or between 0 and $-\pi / 2$ 
according as the polarity of the particle charge, and value of $k x_{0}$, between $-\pi$ and $\pi$. Therefore the ranges of the phase arguments in Eq.(8), (2n-1) $\phi \pm k x_{0}$ and/or $2 n \phi \pm k x_{0}$, exceed the range between $-\pi$ and $\pi$, over which the four-quadrant arctangent can cover as a single valued function. As a result, angles greater than $\pi$ or less than $-\pi$ are folded in the range between $-\pi$ and $\pi$. Then we will have several possible values for $\phi$ when we consider the effect of folding. Table 1 lists these possible values for a few harmonics, where $\phi^{*}$ and $k x_{0} *$ are the four quadrant angles directly obtained from DFT analysis. (See Appendix A.) Note that $\phi^{*}$ and $k x_{0}^{*}$ are determined for each harmonic and not necessarily common to different harmonic modes. This table indicates that when $\phi^{*}$ is in the range between $-\pi / 2$ and 0 for the fundamental mode we will have two set of solutions, i.e., $\left(\phi^{*}, k x_{0}{ }^{*}\right)$ and $\left(\phi^{*}+\pi, k x_{0}{ }^{*} \pm \pi\right)$. The double sign, \pm , should be so selected that $k x_{0}{ }^{*} \pm \pi$ lies in the range from $-\pi$ to $\pi$. In this way we have two different values for $\phi$, which differ by $\pi$ each other, and we cannot discriminate the polarity of charge. But if we use two harmonics, say the fundamental and the second, we will have four sets of solutions for $\phi$ and $k x_{0}$. Then we can find a unique set of $\phi$ and $k x_{0}$, which is common to both the harmonics. As $J_{\mathrm{n}}(k A)$ has zeros, every harmonic vanishes at some values of $k A$ specific to the order of the Bessel function, and therefore, the table is prepared for several harmonics. Here we have to note that two harmonics should be selected such that one is the odd mode and the other is the even mode, otherwise we cannot find a unique solution. Although this method was not used in the experiment because the polarity of droplet was known from the charging condition, a computer simulation confirmed the method. A detailed explanation on the table will be given in Appendix A. 


\subsection{Size and charge measurement}

As is well known, we can determine the particle radius from the phase lag. But we know also that the Stokes law for the drag of a sphere in steady motion is not necessarily applicable to the drag of an oscillating sphere even when the Reynolds number is infinitesimally small. Then a simplified Basset type solution for the drag of an oscillating sphere (Hinze, 1959) is used here.

The drag force, $Z$, exerted on a sphere of radius $r_{\mathrm{p}}$ in a stationary oscillation at a velocity of $A \omega_{\mathrm{p}} \sin \left(\omega_{\mathrm{p}} \mathrm{t}\right)$ can be written as:

$$
Z=6 \pi v \rho_{\mathrm{f}} r_{\mathrm{p}} A \omega_{\mathrm{p}}\left\{\left(1+\frac{\lambda}{\sqrt{2}}\right) \sin \left(\omega_{\mathrm{p}} t\right)+\left(\frac{\lambda}{\sqrt{2}}+\frac{\lambda^{2}}{9}\right) \cos \left(\omega_{\mathrm{p}} t\right)\right\}, \quad \lambda=r_{\mathrm{p}} \sqrt{\omega_{\mathrm{p}} / v}
$$

where $v$ is the kinematic viscosity of surrounding fluid, $\rho_{\mathrm{f}}$ the density of fluid, and $\lambda$ the size parameter defined above. This can be derived from the Navier-Stokes equations for incompressible flow by neglecting the non-linear convection term, and therefore, the amplitude should be very small. It reduces to Stokes law when $\lambda$ approaches zero.

We incorporate Eq.(10) into the equation of motion, in which we assume that the phase of electric field is advanced by $\psi$ with respect to particle velocity, $A \omega_{p} \sin \left(\omega_{p} t\right) . \psi$ is of course related to $\phi$ by $\psi=\phi-\pi / 2$.

$$
\frac{4 \pi r_{\mathrm{p}}^{3} \rho_{\mathrm{p}}}{3} \frac{\mathrm{d}\left\{A \omega_{\mathrm{p}} \sin \left(\omega_{\mathrm{p}} t\right)\right\}}{\mathrm{d} t}+Z=q E_{0} \sin \left(\omega_{\mathrm{p}} t+\psi\right)
$$

where $\rho_{\mathrm{p}}$ is the particle density, $q$ the electrostatic charge of the particle, and $E_{0}$ the field strength 
for particle oscillation. From this equation, we have

$$
\begin{aligned}
& r_{\mathrm{p}}=\frac{3 \rho_{\mathrm{f}} \sqrt{v}}{2\left(2 \rho_{\mathrm{p}}+\rho_{\mathrm{f}}\right) \sqrt{2 \omega_{\mathrm{p}}}}\left\{3(\tan \psi-1)+\sqrt{9+\left\{9 \tan \psi+16\left(\rho_{\mathrm{p}} / \rho_{\mathrm{f}}\right)-10\right\} \tan \psi}\right\} \\
& q=\frac{6 \pi \rho_{\mathrm{f}} v r_{\mathrm{p}} A \omega_{\mathrm{p}}}{E_{0} \cos \psi}\left\{1+r_{\mathrm{p}} \sqrt{\frac{\omega_{\mathrm{p}}}{2 v}}\right\} .
\end{aligned}
$$

For the measurement in normal air, the discrepancy between $r_{\mathrm{p}}$ thus calculated and that calculated from conventional Stokes low is within $\pm 5 \%$ when $10^{\circ} \leq \psi \leq 80^{\circ}$ (or $-100^{\circ} \geq \psi \geq-170^{\circ}$ ) for $\rho_{\mathrm{p}}=1 \mathrm{~g} / \mathrm{cm}^{3}$. As for the charge, it ranges from $-3 \%$ to $19 \%$. These discrepancies decrease with increasing $\rho_{\mathrm{p}}$ such that they are inversely proportional to the square root of $\rho_{\mathrm{p}}$. Usually such a small difference may be ignored, but the systematic dependency of the discrepancies on $\psi$ may bring about a false trend and can give rise to substantial misunderstanding when we deal with, e.g., the time variations in size and/or charge of an evaporating droplet.

\section{Experimental setup and measured results}

Fig.8 is the schematic diagram of the measuring system. A $30 \mathrm{~mW}$ He-Ne laser was used to form stationary interference fringes at the center of the quadrupole electrodes to measure the particle motion in the direction perpendicular to the parallel plate electrodes. The spacing of the fringe planes was $9.5 \mu \mathrm{m}$, and the diameter of the measurement volume was adjustable with a collimator but usually $2 \mathrm{~mm}$. Droplets to be measured were generated by a vibrating orifice aerosol generator (TSI model 3050). To charge the droplet by induction charging, a small ring 
electrode applied a dc electric field to the tip of the liquid jet as it leaves from the micro orifice of the generator. The voltage to the electrode could be adjusted within $\pm 6 \mathrm{kV}$ in response to the required droplet charge and its polarity determined the polarity of the charge. The liquids for droplets were alcohol solutions of low- or non-volatile liquids to be tested. Alcohol was completely evaporated in an electrostatic curtain, which consisted of 16 metal rods forming a cylindrical cage. A high ac voltage was applied to every second rod and the rest of the rods were grounded. The ac electric field between the rods repulsed the charged droplet inside the cage by the electrodynamic effect. A shutter placed on the top of the measurement cell was opened for a short moment to lead the charged droplets into the upper section of the cell. The trapping ac field prevented the charged particles flowing down into the measuring section, because the ac electric field formed by the upper rod electrode of the quadrupole electrode assembly gave rise to electrodynamic repulsion on the charged particles from the rod electrode. A very short intermission of the trapping ac voltage allowed some charged particles to enter into the measuring section. This process was repeated until a single charged particle was incidentally trapped in the measuring section. When a single particle was successfully trapped, the LDV signal took the form of an FM wave without amplitude modulation like the one shown in Fig.4. Once a single droplet was trapped, the measurement could be repeated every 0.3 second. Further explanation on the operation of the apparatus may be found elsewhere (Nakajima and Sato, 2003).

Another thing that should be pointed out is the use of spatial filter located at the focal plane 
of the light-collecting lens of the optical receiver. As is well known, the visibility of the beat signal deteriorates when the particle size becomes larger than the fringe spacing (Farmer, 1972 and 1974). Roberds (1977) proposed a formula for the signal visibility, and showed that the visibility can be improved with the use of small light collection aperture at the sacrifice of sensitivity. In this experimental apparatus, a hyperbolic slit shown in Fig.8 was used as a spatial filter (or laser beam stop) at the focal plane of the optical receiver to improve the visibility. The spatial filter made the visibility greater than 0.5 for particles smaller than $30 \mu \mathrm{m}$ in diameter for a fringe spacing of $9.5 \mu \mathrm{m}$ at the minimum sacrifice of sensitivity.

\subsection{Accuracy of the size and charge measurement}

To examine the accuracy of the measuring system, the time variations in size and charge of DnOP

(Di-n-Octyl Phthalete) droplets were measured. An example is shown in Fig.9. The upper and the lower bands of data points in the figure, respectively, are for droplet diameter and for the charge.

These were recorded every 2 seconds for 10 hours. As DnOP is a non-volatile liquid in normal room condition, the size of droplet is expected to remain constant. In fact, the diameter was fairly stable and it was calculated to be $6.873 \pm 0.030 \mu \mathrm{m}$ (or less than $0.5 \%$ error in size) with a confidence coefficient of $99 \%$. As the major source of the error in size measurement is the error in phase measurement, Eq.(12) is differentiated with respect to $\psi$ to obtain the sensitivity of the size to the phase. We found the sensitivity is the lowest or the optimum at $\psi=45^{\circ}$. Fig. 10 shows the sensitivity relative to the optimum. The data in Fig.9 was collected at the optimum $\psi\left(=45^{\circ}\right)$. 
Since we may expect the error in phase measurement by the Fourier analysis is independent of $\psi$ in principle, we see from Fig.10 that the error for range of $\psi$ between $15^{\circ}$ and $75^{\circ}$ would be less than twice that at the optimum. Therefore we can use a fixed frequency for fairly wide range of size. The size ratio measurable with a fixed frequency will be 4 if we admit $1 \%$ error in size with a $99 \%$ confidence coefficient for the present apparatus.

As for the droplet charge, it decreased gradually due to recombination with ions from surrounding gas medium, local electron avalanches, and some other possible causes such as evaporation of ions. In Fig.9, we found a relatively stable interval shown by white arrow, and the average was taken over the interval to give $52.89 \pm 0.46 \mathrm{fC}$ with a confidence coefficient of $99 \%$. Although sensitivity analysis was not performed for charge measurement, we may expect an error of less than $3 \%$ if size was measured within $1 \%$ error.

Since this apparatus can repeat the measurement every 0.3 second for a single particle, the error will be reduced by a factor of 0.1 by taking the average of measured values over a period of 30 seconds, or over 100 data: as is well known, the error or the standard deviation of an average is inversely proportional to the square root of the number of data averaged. Therefore the accuracy of measurement can be improved to higher level if the time variations are not very quick in comparison with the averaging period. As the digitizer ( $\mathrm{AD}$ converter) used in this apparatus was not a state-of-the-art equipment, and it caused considerable phase error; the use of high precision digitizer is expected to improve the accuracy. 


\subsection{Rayleigh instability of charged droplets}

As a charged droplet evaporates, the charge density on the droplet surface gets higher and higher.

When the Coulombic repulsion overcomes the cohesion due to surface tension, the droplet disrupts. The critical charge for the Coulombic fission is called the Rayleigh limit. For inviscid conductive liquids, the critical charge was theoretically predicted to be (Rayleigh, 1882, Hendricks and Schneider, 1963):

$$
\left|Q_{R L}\right|=2 \pi \sqrt{2 \varepsilon \sigma D_{p}^{3}}
$$

where $\varepsilon$ is the permittivity of surrounding gas medium $\left(\approx 10^{-9} / 36 \pi \mathrm{F} / \mathrm{m}\right.$ for air), $\sigma$ the surface tension and $D_{\mathrm{p}}$ the droplet diameter. This formula has been widely used in various scientific fields, even in nuclear fission (e.g., Chandezon et al., 2001). A number of experiments have been carried out to inspect the mechanism. But the mechanism of the Rayleigh disruption has not been well understood yet.

Fig.11 is an example of such measurements with the present apparatus for evaporating DBP (Di-Buthyl Phthalate) droplets. The diameter of the DBP droplet reduced from $11 \mu \mathrm{m}$ to 4 $\mu \mathrm{m}$ during the evaporation. But the reduction was not continuous and we found several occurrences of Rayleigh disruption. The values attached at the moments of discontinuous size reductions are the fractions of volume loss at the disruption. The charge remained virtually constant until the Rayleigh disruption occurred. The percentages attached to the charge variation are the critical charges relative to the Rayleigh limit, i.e., $\left|Q_{\mathrm{RL}}\right|$ given in Eq.(14), and the charge loss fractions at the disruption. We see from the figure that the Rayleigh fission occurs at about 
$95 \%$ of the Rayleigh limit for this case. In this experiment on DBP, the Rayleigh fission was accompanied by $10 \sim 20 \%$ mass loss and about $25 \%$ charge loss. This corresponds to so-called “rough fission” mode (Fernández de la Mora, J., 1995) that emits a few but relatively large daughter droplets. This mode was reported by Abbas and Latham (1967) for water, aniline and toluene, and by Taflin et al. (1989) for DBP.

Fig.12 shows another mode, namely, the "fine fission" mode, in which the mass loss is as small as $1 \%$ and the charge loss is $10 \sim 20 \%$. These data were collected for a droplet of pentadecane. The loss of charge was $7 \sim 13 \%$, and the loss of mass was so small that the discontinuity in the size variation was rather hard to notice without magnification as shown in the figure. Numerically, it was found to be $0.7 \sim 1 \%$. The data reported by Taflin et al. (1989) suggested that hexadecane and heptadecane also belong to this mode. Using high-speed microscopic images, Duft et al. (2003) succeeded to take detailed photos of Rayleigh fission of a charged ethylene glycol droplet, which deformed to an ellipsoid with two sharp tips on its poles and finally ejected fine liquid jets from both tips. The charge loss was reported to be $33 \%$, and the mass loss, $0.3 \%$. At the same time, they reported that about 100 daughter droplets of $1.5 \mu \mathrm{m}$ in diameter were expelled from a $24 \mu \mathrm{m}$ parent droplet, which implied a mass loss of $2.4 \%$. The data in a previous paper by Duft et al. (2002) showed that the charge loss was on the order of $20 \%$ on the average. These facts suggest that ethylene glycol presumably disrupts in the fine fission mode. The disruption observed in the experiments mentioned above occurred at 90 $\sim 100 \%$ of the Rayleigh limit except for data by Taflin et al., which showed somewhat too low 
values for all liquids they tested.

Richardson et al. (1989) reported an extreme case of the "fine fission" mode for a droplet of sulfuric acid. The charge loss was about $50 \%$ but no mass loss was observed. The disruption occurred at $92 \%$ of the Rayleigh limit on the average, though their measured data for charge were rather scattered. Fig.13 shows another example of the extreme case measured by the present apparatus for glycerol droplet. The charge losses were comparatively large but no discontinuous reduction in size was seen. Experiments were repeated many times for glycerol droplets, but discontinuous size reduction was never observed even in the occasional cases when the charge loss exceeded $50 \%$. Another observation made was that the discharge occurred at around $80 \%$ of the Rayleigh limit, which was appreciably lower in comparison with other liquids. As the Rayleigh limit for glycerol is high due to its high surface tension, the field strength at the droplet surface could exceed $60 \mathrm{MV} / \mathrm{m}$. According to the theory of Fernández de la Mora (1996), high electrical conductivity of liquids favors the "fine fission" mode, but unlike sulfuric acid, glycerol is not a high conductive liquid. For these reasons, it was quite reasonable to suppose that the result shown in Fig.13 might indicate an occurrence of the electric breakdown, which brought the charge limit of small spherical solid particles in normal air. Then the effects of polarity and medium gas on the relationship between the maximum charge and droplet size were examined, because the breakdown depends on them while the Rayleigh fission does not. A highly insulating gas (i.e., $\mathrm{SF}_{6}$ gas) was used for the medium gas and an appreciable increase in the maximum charge was expected. The measurements were carried out for both polarities. 
An example of the comparison with the corresponding results in normal air is illustrated in Fig.14, in which the charge is plotted against the size. As is seen in the figure, the charge remained almost constant during the evaporation until it hit on the limit line, which is independent of the medium gases and the polarities. Therefore, we had to conclude that the large losses in droplet charge shown in Fig.13 were not a result of the air breakdown but the disruption of glycerol droplets in the "extremely fine fission” mode. It has not been clarified what happens on the droplet surface in the "extremely fine fission" mode, but probably, the parent droplet ejects a number of very fine daughter droplets, whose charge to mass ratio can be very high within Rayleigh limit. Note that the charge to mass ratio of a droplet at the Rayleigh limit is inversely proportional to $D_{\mathrm{p}}^{1.5}$, i.e., we have $\left|Q_{R L}\right| / m=12 \sqrt{2 \varepsilon \sigma / \rho_{p}^{2} D_{p}^{3}}$ from Eq.(14).

\subsection{Maximum charge sustainable for spherical particles in normal air}

It has been frequently assumed that electric breakdown in normal air occurs at a field strength of around 3MV/m. But the smaller the space of high electric field becomes, the higher the critical field strength. In fact, the observed data for glycerol droplets (e.g., Figs. 13 and 14) indicated that the field strengths at the surfaces of droplets amounted to $50 \mathrm{MV} / \mathrm{m}$ for $16 \mu \mathrm{m}$ in diameter, 62 $\mathrm{MV} / \mathrm{m}$ for $9.8 \mu \mathrm{m}$ and $90 \mathrm{MV} / \mathrm{m}$ for $4.4 \mu \mathrm{m}$, which were much higher than $3 \mathrm{MV} / \mathrm{m}$. Therefore, small particles can carry a larger amount of charge than that is normally expected. There have been several theoretical predictions (Harper, 1967, Pedersen, 1967, Elghazaly and Castle, 1987, Pedersen, 1989, Crowley, 1991, Nakajima and Sato, 1999a,b) for the maximum charge of 
spherical solid particles sustainable in normal air. Fig.15 shows them with the Rayleigh fission data for glycerol droplets in normal air in a bold broken line. The papers by Pedersen (1967, 1989) did not actually refer to the breakdown around a charged isolated particle. The curves labeled "Pedersen" in Fig.15 were calculated by the author and his colleague (Nakajima and Sato, 1999a,b) after Pedersen's novel work. Fig.15 shows that the calculated maximum charge depends on not only the size of particle but also on the polarity of the charge, which is denoted by the sign in parentheses. Here we have to note that the critical values for the breakdown must be higher than the observed data for glycerol droplets shown in the bold gray dotted line, because the data were limited by the Rayleigh fission. In this sense, the predictions by Crowley(1991) and Harper(1967) would give too low values.

All predictions for negative charge, except for Crowley's, were based on the theory of Townsend (e.g., Engel, 1965). The differences among them largely came from the differences in the estimation of the coefficients for the ionization of the medium gas and for the secondary electron emission of the cathode. Since very few data were available for very high electric field, these estimations would be plausible only for the particles larger than $100 \mu \mathrm{m}$. Nakajima and Sato (1999a) searched for the data usable in very high field strength, and proposed a formula for the maximum charge, which is expected to be valid for the particles larger than $10 \mu \mathrm{m}$ in diameter. The contribution of this apparatus to their discussions may be found elsewhere (Nakajima and Sato, 1999a, b). As the papers were published in Japanese, a brief explanation is given in the Appendix B together with the Townsend theory. 


\section{Discussions}

The present apparatus can measure the time variation in the size and charge of a single droplet repeatedly by the use of a simplified trapping electrode. The apparatus can be constructed with commonly available instruments and without special skill. The advantage of the present method is high accuracy of the size and charge measurements. If the time variation is not too fast, we can improve the accuracy by taking the moving averages of the measured results. But to ensure its accuracy, it is necessary to take into consideration the following points:

1) The amplitude of the particle oscillation should be as small as possible. This is because the hydrodynamic relation between the size and the phase lag is appropriate only for infinitesimally small amplitude. In this experiment, the amplitude was selected to be around $5.2 \mu \mathrm{m}$.

2) The phase error of the digitizer should be compensated as accurately as possible. In this experimental apparatus, the phase error was compensated within $\pm 0.05^{\circ}$ at the DFT processing stage for the frequency range of 0.4 to $6.4 \mathrm{kHz}$. On the other hand, the sensitivity error and non-linearity of the digitizer will not be vital factors, because the DFT analysis for the phase and amplitude measurements is not very sensitive to them. Further, the sampling rate of digitizer may not be necessarily very high. A simulation showed that it is sufficient to select the sampling frequency of the digitizer to be 50 times the frequency of the driving ac voltage when the amplitude of particle is less than or the order of the fringe size. So a digitizer with $1 \mathrm{MHz}$ maximum sampling frequency will suffice for usual purposes. 
3) The length of a data record for the DFT analysis should cover several tens cycles of the oscillation of the droplet. As a result, it will take at least several tens ms for a single measurement. In this experiment, one record covered 85 cycles and it took about 300 ms including the time for DFT analysis. The use of a faster computer may shorten the time to $100 \mathrm{~ms}$. This will be preferable for the moving average technique. At any rate, however, this method is not suitable for high-speed measurement in contrast to the E-SPART analyzer.

4) The FFT algorithm is not recommended to use because it analyzes the signal at predetermined frequencies while the present method requires Fourier coefficients at arbitrarily given frequencies corresponding to $n \omega_{\mathrm{p}} \pm k u(\mathrm{n}=0,1, \ldots \ldots, 6)$. Note that $\omega_{\mathrm{p}}$ and $u$ are not given in advance but are to be determined accurately by the DFT analysis. Application of hannig window (Blackman and Tukey, 1958) to raw data and linear interpolation between the discrete data points were shown to be very effective to improve the reliability of DFT analysis.

5) The diameter of the laser beams should be selected to be $2 \mathrm{~mm}$ or larger when droplets as large as $15 \mu \mathrm{m}$ in diameter are to be measured. This is because the droplet starts to fall by gravity when the trapping electric field is intermitted during the data acquisition period.

6) As droplet evaporates, the intensity of scattered light weakens much. So it is recommended to use a 12-bit digitizer or an automatic sensitivity control system for the optical receiver. In connection to this, some effective means for improving the signal visibility should be prepared for droplets larger than the fringe spacing. The use of a small round aperture at the optical receiver (Roberds, 1977) may not be very advantageous in the sense of sensitivity. Instead, a 
hyperbolic slit as shown in Fig.8 is recommended to use. The droplet size actually measurable by this experimental apparatus was from $18 \mu \mathrm{m}$ to $3 \mu \mathrm{m}$ with $30 \mathrm{~mW}$ He-Ne laser. When the laser beams were focused to $0.8 \mathrm{~mm}$ in diameter, the minimum measurable size was about $1 \mu \mathrm{m}$ but the accuracy of the measurements was much deteriorated because optimum driving frequency for this size became 10 times higher than that available for the apparatus.

7) Unlike the E-SPART analyzers, the present method is not usable when the droplet charge is too small because the particle cannot be trapped by electrodynamic means. Application of high voltage to the trapping electrodes is limited by the electric breakdown of medium gas, which discharges particle charge by recombination with ions generated by the breakdown. For the present experimental apparatus, the minimum charge is around $5 \mathrm{fC}$ for a particle of $6 \mu \mathrm{m}$ in diameter. For larger particles, lowering the frequency of trapping electric ac field from $700 \mathrm{~Hz}$ to $100 \mathrm{~Hz}$ will be effective if necessary. This is because the trapping force can be strengthened by using lower frequency for large particles as we may see from the first term in the right hand side of Eq.(4).

In the present method, a suitable dc component is superimposed on the ac driving voltage to give the charged droplet a constant velocity component such that $u=\omega_{\mathrm{p}} / 4 k$. This makes the measurements accurate, but the dc bias has to be regulated in response to the charge and size of the droplet. Therefore, we had to seek a suitable dc bias by trials until we succeeded to start the automatic control system by giving incidentally an approximate value to the desired bias. Once the system started, the dc bias was automatically adjusted by referring to the preceding 
measurement. This initial procedure is time consuming and will be a severe defect when we wish to make measurements on volatile liquid like water. To make use of the present apparatus in the areas mentioned in the introduction of this paper, the following improvements may be recommended.

1) If we use moving interference fringes instead of adding a constant velocity component to the droplet motion, the initial procedure can be skipped. Bragg cells, which modulate laser light waves with a radio frequency of typically $40 \mathrm{MHz}$, have been widely used in LDV technique to move the fringes at a constant velocity by shifting the frequency of one of two coherent laser beams. For the present method, however, the amount of frequency shift required is only a quarter the frequency of driving ac voltage for droplet oscillation, which is extremely low for a Bragg cell frequency shifter. This difficulty may be resolved by using a combination of two frequency shifters, which can achieve low amount of shift, namely from dc to kHz. No revisions in the data processing algorithm will be required for this improvement.

2) Another improvement to shorten the time taken to initiate the measurement is to use electrostatic guide and one-by-one droplet feed gate. As suggested before, trapping a single droplet in the measurement volume required a rather tedious and time-consuming procedure. Masuda et al. (1980) proposed a quadrupole rail guide and electrostatic gate system to feed deuterium oxide (heavy water) pellet one-by-one into a fusion reactor in high vacuum. If such a system becomes available for the present apparatus in the future, we can carry out measurements with volatile matter more easily, and furthermore, we will be able to perform experiments in 
reduced gas pressure, which are expected to yield essential and valuable information for the theory of electric breakdown and electron avalanche in strongly non-uniform electric fields. The results will contribute valuable data and new approach to the micro discharging process, which is now getting more and more important in the industrial applications.

\section{Conclusions}

It was shown that a simple LDV system with its sensing volume located within a quadruple electrode assembly can be used to measure the time variation of the size and charge of a single evaporating droplet. A mechanism for trapping charged particles in a quadrupole electric field was developed to simplify the use of an electrodynamic trap. A new approach to a signal processing system for the LDV signals for size and charge measurements was developed to improve the accuracy of size and charge measurements.

The accuracy of the measurements was discussed using actual experimental data: the errors in size and in charge, respectively, were approximately $0.1 \%$ and $0.3 \%$ when the data were collected and averaged for a period of 30 seconds.

The measurement system can be used to study Rayleigh instability of charged droplets. Experimental data were presented for low volatile liquids. Three types of the Rayleigh fission were observed. One is the "rough fission" mode, in which the droplet mass loss at each fission is on the order of $10 \%$ with a charge loss of 20 to $30 \%$. The "fine fission" mode is classified into two types. In the first type, both the mass and charge losses, in particular the former, are 
appreciably smaller than those in the "rough fission" mode. In the second type, the charge loss is very high (30 50\%) while the mass loss is imperceptible, possibly caused by ejection of very fine charged daughter droplets and not by air breakdown around the droplet, because the experiments in highly insulating gas $\left(\mathrm{SF}_{6}\right)$ showed the same discharging characteristics as in air.

The charge limit due to electric breakdown of air around a small solid spherical particle was considered. Several existing predictions for the limit were compared with the data for glycerol droplets, for which it was shown that the charge loss was caused by Rayleigh fission. As no Rayleigh fission occurs for solid particles, the predicted limit for solid particles should be higher than the observed limit for glycerol droplets. Nevertheless, some predictions were below the glycerol data showing that they may not be acceptable. For negatively charged particles, a prediction based on the Townsend theory was briefly explained (in Appendix B) to propose a reasonable correlation for the charge limit due to the electron avalanches in normal air. As for positively charged particles, the limit is still left unclarified, but the correlation for negative charge may be applicable to positively charged particles because the experiment in argon gas showed no significant difference between the two polarities.

\section{Acknowledgments}

This work has been performed under continuous stimulations from Prof. T. Sato of Hokkaido institute of Technology. Some parts of this paper were results of collaboration with him. The author also would like to express his deep gratitude to the reviewers for their help to revise the 
manuscript; in particular, one reviewer kindly corrected the manuscript with helpful advice and thoughtful comments.

\section{Appendix A Explanation of Table 1 for polarity discrimination}

From Eq. (8), we have for the leading terms of the beat signal:

$$
\begin{aligned}
\mathrm{i}_{p}= & B J_{0}(k A) \cos \left(k u t+k x_{0}\right) \\
& +B J_{1}(k A)\left[\cos \left\{\left(\omega_{\mathrm{p}}+k u\right) t-\left(\phi-k x_{0}\right)\right\}-\cos \left\{\left(\omega_{\mathrm{p}}-k u\right) t-\left(\phi+k x_{0}\right)\right\}\right] \\
& +B J_{2}(k A)\left[\cos \left\{\left(2 \omega_{\mathrm{p}}+k u\right) t-\left(2 \phi-k x_{0}\right)\right\}+\cos \left\{\left(2 \omega_{\mathrm{p}}-k u\right) t-\left(2 \phi+k x_{0}\right)\right\}\right] \\
& +B J_{3}(k A)\left[\cos \left\{\left(3 \omega_{\mathrm{p}}+k u\right) t-\left(3 \phi-k x_{0}\right)\right\}-\cos \left\{\left(3 \omega_{\mathrm{p}}-k u\right) t-\left(3 \phi+k x_{0}\right)\right\}\right] \\
& +B J_{4}(k A)\left[\cos \left\{\left(4 \omega_{\mathrm{p}}+k u\right) t-\left(4 \phi-k x_{0}\right)\right\}+\cos \left\{\left(4 \omega_{\mathrm{p}}-k u\right) t-\left(4 \phi+k x_{0}\right)\right\}\right]
\end{aligned}
$$

The principal value of arctangent is defined in the range between $-\pi / 2$ and $\pi / 2$, but in DFT

analysis we use the four-quadrant arctangent, which gives values from $-\pi$ to $\pi$. Hence we can determine a unique value of $k x_{0}$ from the 0 -th mode, i.e., the first term in the right hand side of Eq. (A1). Note here that we have to check the sign of the Bessel function, $J_{\mathrm{n}}(k A)$ in response to the value of $k A$ because its negative sign makes the phase shift by $\pi$. From the fundamental mode, i.e., the second term, we may obtain two phase components, $A_{1}=\phi+k x_{0}$ and $S_{1}=\phi-k x_{0}$, by DFT. As their values may be folded in the range from $-\pi$ to $\pi$, it is better to express them to be $A_{1}{ }^{*}=\phi^{*}$ $+k x_{0} *$ and $S_{1}^{*}=\phi^{*}-k x_{0} *$, which are obtainable directly from DFT complex coefficients. Thus we have $\phi^{*}=\left(A_{1}{ }^{*}+S_{1}{ }^{*}\right) / 2$ and $k x_{0} *=\left(A_{1}^{*}-S_{1} *\right) / 2$, and then two sets of possible solution for $\phi$ and $k x_{0}$ can be found with the aid of Table 1 . As only one of the sets has the same value for $k x_{0}$ as that 
calculated from the 0-th mode, we can select the proper set and determine the correct value for $\phi$, which make us possible to discriminate the polarity of the charge. However, it should be noted here that $J_{0}(k A)$ has zeros at $k A=2.405,5.520,8.654$ and so on, and also $J_{1}(k A)$ has zeros at $k A=3.832,7.016,10.173$ and so on. Therefore, it is not always possible for us to determine the values by using the 0 -th and the fundamental modes. To avoid such difficulty, Table 1 includes harmonics up to 4th mode. In this Appendix, the derivation of the equations listed in Table 1 is explained to make the method established. The third mode is exemplified here because it seems the most complicated case in the table.

Fig.A1 shows the map of angle corrections required for $A_{3} *$ and $S_{3} *$ to obtain the correct values for $A_{3}\left(=3 \phi+k x_{0}\right)$ and $S_{3}\left(=3 \phi-k x_{0}\right)$ when the values of $\phi$ and $k x_{0}$, respectively, are given by the abscissa and the ordinate of the figure. The calculated values for $A_{3}$ and $S_{3}$ by DFT are denoted by $A_{3}{ }^{*}$ and $S_{3}{ }^{*}$, which may be folded in the range between $-\pi$ and $\pi$. We find there are three cases when $\pi>\phi \geq 2 \pi / 3$ as shown in the right side of the figure. In the triangular section denoted by 'case 1 ', the inequalities, $-3 \phi+5 \pi>k x_{0}>-3 \phi+3 \pi$ and $3 \phi-\pi>k x_{0}>3 \phi-3 \pi$, hold. It follows that $5 \pi>A_{3}>3 \pi$ and $3 \pi>S_{3}>\pi$, and therefore, $A_{3}=A_{3}{ }^{*}+4 \pi$ and $S_{3}=S_{3} *+2 \pi$. Then if $\phi$ and $k x_{0}$ are in this triangle, $\phi=\left(A_{3}+S_{3}\right) / 6=\left(A_{3}{ }^{*}+S_{3}{ }^{*}+6 \pi\right) / 6=\phi^{*}+\pi$ and $k x_{0}=\left(A_{3}-S_{3}\right) / 2=$ $\left(A_{3}{ }^{*}-S_{3}{ }^{*}+2 \pi\right) / 2=k x_{0}{ }^{*}+\pi$. As $\phi$ is supposed in the range $\pi>\phi \geq 2 \pi / 3$ then $0>\phi^{*}>-\pi / 3$. This result is tabulated in Table 1 as case (b) for $-\pi / 3<\phi^{*}<-\pi / 6$ and case (d) for $-\pi / 6<\phi^{*}<0$. In the diamond shaped section denoted by 'case 2', the similar discussion as above yields $A_{3}=A_{3}{ }^{*}+2 \pi$ and $S_{3}=S_{3} *+2 \pi$. Then $\phi=\left(A_{3}+S_{3}\right) / 6=\left(A_{3} *+S_{3} *+4 \pi\right) / 6=\phi^{*}+2 \pi / 3$ and $k x_{0}=$ 
$\left(A_{3}-S_{3}\right) / 2=\left(A_{3} *-S_{3} *\right) / 2=k x_{0} *$. These results are reflected in the table as the case (c) for $-\pi / 6$

$<\phi^{*}<0$, the case (b) for $0<\phi^{*}<\pi / 6$ and the case (d) for $\pi / 6<\phi^{*}<\pi / 3$. Note here that the

section for $\pi / 3<\phi<\pi / 2$ in the diamond is excluded because $\phi$ never lies in this range. As for the

'case 3', we have $\phi=\left(A_{3}+S_{3}\right) / 6=\left(A_{3}{ }^{*}+S_{3}{ }^{*}+6 \pi\right) / 6=\phi^{*}+\pi$ and $k x_{0}=\left(A_{3}-S_{3}\right) / 2=$

$\left(A_{3}{ }^{*}-S_{3}{ }^{*}-2 \pi\right) / 2=k x_{0} *-\pi$. This yields the same results as the 'case 1' except for $k x_{0}=k x_{0} *-\pi$,

but $k x_{0}=k x_{0} * \pm \pi$ gives the same $k x_{0}$ if $k x_{0}$ is selected to be an angle in the four quadrant.

As suggested in the main text, we have to select two harmonics such that one is the odd

mode and the other is the even mode. The reason is explained as below:

Suppose that $\phi$ and $k x_{0}$ for the case (a) in the second mode for $-\pi / 2<\phi^{*}<0$ agree with

those for the case (a) in the fourth mode for $-\pi / 4<\phi^{*}<0$, then the case (b) for the second mode automatically gives the same values of the case (c) for the fourth mode. (Remember that $\phi^{*}$ and $k x_{0} *$ are not always common to different modes.) As a result, we have two values of $\phi$, which differ by $\pi$ each other, and we cannot discriminate the polarity of the charge. Further inspection of Table 1 shows that we have to select modes for the discrimination of the polarity in such a way that mentioned above.

\section{Appendix B Semi-empirical equation for the maximum charge}

To begin with, the theory of Townsend (e.g., Engel, 1965) is outlined for the case of negatively charged particle. An electron, which can be generated by some causes such as the gamma process described later and ionization by natural radiation, is accelerated by the electric field formed by 
the particle charge, and undergoes collisions with neutral gas molecules. If the electron gains enough energy from the electric field before the collision, the electron sometimes liberates one electron from neutral gas molecular leaving a cation. The number of electrons thus liberated per unit length that a single electron goes through is called the ionization coefficient, $\alpha$. It depends on the gas properties and is a function of the electric field strength, $E$, and the ambient pressure, $p$ (or more correctly, the number of gas molecules per unit volume). Many forms of approximation for $\alpha$ are possible (Schumann, 1923), but usually it takes the form of $\alpha=a p \exp (-b p / E)$. However, we adopted the following form for better approximation in wide range of field strength. The following numerical correlation was obtained for dry air from the data collected by Dutton (1975):

$$
\frac{\alpha}{p_{20}}=132.1\left\{1+\left(\frac{937.8}{E / p_{20}}\right)^{3}+\left(\frac{473.3}{E / p_{20}}\right)^{6}\right\} \exp \left\{-\frac{3343}{E / p_{20}}\right\}
$$

where $p_{20}$ is the reduced pressure that means the pressure at which the density of gas at $20{ }^{\circ} \mathrm{C}$ is equal to the density of actual gas surrounding the electrode. As Eq.(B1) is an empirical equation, we have to use specified units, namely, $\mathrm{kPa}$ for pressure, $\mathrm{V} / \mathrm{cm}$ for electric field and $\mathrm{cm}^{-1}$ for $\alpha$. The range of $E / p_{20}$ in the original data was from 500 to $7000 \mathrm{~V} /(\mathrm{cm} \mathrm{kPa)}$, where Eq.(B1) gives very close approximation to the numerical data.

The total number of electrons bred by a single electron starting from the surface of the negatively charged particle (or the cathode) of radius $r_{\mathrm{p}}$ will be 


$$
n_{\mathrm{e}}=\exp \left(\int_{r_{\mathrm{p}}}^{r_{0}} \alpha \mathrm{d} r\right)
$$

where $r_{0}$ is the radius coordinate at which the electric field becomes too weak to make ionizing collision. As a result, $\left(n_{\mathrm{e}}-1\right)$ cations drift back to the cathode (i.e., the surface of negatively charged particle). On impact with the cathode, each cation will release a secondary electron with a probability, $\gamma$, the secondary electron emission coefficient. This is called the gamma process. Therefore the discharge will be self-sustaining if $\left(n_{\mathrm{e}}-1\right) \gamma \geq 1$, and the following equality holds at the critical point for breakdown:

$$
\gamma\left\{\exp \left(\int_{r_{\mathrm{p}}}^{r_{0}} \alpha \mathrm{d} r\right)-1\right\}=1
$$

This is the Townsend criterion for self-sustaining discharge.

Unfortunately, no suitable data for $\gamma$ applicable to wide range of field strength were reported in literatures. But if we know $\alpha, \gamma$ can be estimated from the Paschen curve, which relates the breakdown voltage, $V_{\mathrm{bk}}[\mathrm{V}]$, for parallel plate electrodes to the gap size $d$ [cm]. The extensive data for dry air collected by Wind (1974) can be approximated closely by

$$
V_{\mathrm{bk}}=\left(247.5 p_{20} d+649 \sqrt{p_{20} d}\right)\left\{1+\frac{0.0512}{p_{20} d}+\left(\frac{0.01572}{p_{20} d}\right)^{2}+\left(\frac{0.02784}{p_{20} d}\right)^{4}\right\}
$$

For an arbitrarily supposed value of $p_{20} d[\mathrm{kPa} \mathrm{cm}]$, we can calculate $V_{\mathrm{bk}} /\left(p_{20} d\right)$ or $E_{\mathrm{bk}} / p_{20}$ from this equation representing the Paschen curve. Then $E_{\mathrm{bk}} / p_{20}$ thus obtained is substituted into Eq.(B1) to obtain $\alpha / p_{20}$, which is multiplied by $p_{20} d$ to give $\alpha d$. Since the integral in Eq.(B3) can 
be written as $\alpha d$ for parallel plate electrodes, we have for $\gamma$ from Eq.(B3):

$$
\gamma=1 /\{\exp (\alpha d)-1\}
$$

This calculation was repeated for various values of $p_{20} d$ to obtain a correlation between $\gamma$ and $E_{\mathrm{bk}} / p_{20}$. The electric field around a particle of radius, $r_{\mathrm{p}}$, with a charge, $q$, is $E=q / 4 \pi \varepsilon r^{2}$ at the radius coordinate, $r,\left(r>r_{\mathrm{p}}\right)$. For a given reduced pressure, we can calculate $\alpha$ from Eq.(B1) as a function of $r$ to evaluate the integral in Eq.(B3). And further, to estimate $\gamma$ for $E_{\mathrm{bk}} / p_{20}$ from the correlation mentioned above, we may use the field strength at the particle surface for $E_{\mathrm{bk}}$, because the kinetic energy of cation for the gamma process is largely determined by the field strength near the collision surface. In this way, we can calculate the left-hand side of Eq.(B3) and search for the critical charge that satisfies Eq.(B3) by trials. The numerical results for spherical particles in normal air were approximated by the following equation for the diameter range between $1 \mu \mathrm{m}$ and $1 \mathrm{~cm}$.

$$
q_{\max }=1.17 \times 10^{-4} D_{\mathrm{p}}^{2}\left\{1+2.90 \times 10^{-2} D_{\mathrm{p}}^{-0.54}+4.40 \times 10^{-7} D_{\mathrm{p}}^{-1.4}\right\}
$$

In this equation, the units are Coulombs for the maximum charge $q_{\max }$ and meters for the diameter $D_{\mathrm{p}}$.

The data for the Paschen curve represented by Eq.(B4) were mostly collected for brass electrodes, on which the gamma process took place. It may be questionable if the gamma process really occurs on the surface of negatively charged particle, which is non-metallic material. There are no free electrons on the surface, but there are lots of anions from which electrons may be readily liberated by corresponding energy to electron affinity, which is less than the work 
function of metals. So it is not unlikely for the negatively charged particles to emit electrons when the surface is bombarded by cations.

As for positively charged particles, we cannot use the Townsend theory because a cathode is inevitably necessary to emit secondary electrons by the gamma process. To clarify the corona discharge from a positive point electrode, Hartmann (1984) used the Meek’s theory (1940), of which physical picture is not as clear as that of Townsend. Elghazaly and Castle (1987) extended the theory to spherical particles to obtain the curve shown in Fig.15 in the main text. However, Hartman used an approximation for field intensity near the point electrode. As his approximation turned out to be inappropriate for small spherical electrode, it was modified to an exact one for spheres (Nakajima and Sato, 1999b). In Fig.15, the result is shown by the curve labeled “modified”, which seems rather unreasonable.

We need some experimental results that support the above theoretical consideration on electric breakdown around a small particle. As no evidence of breakdown was observed in the experiments in air, an experiment was carried out in argon gas, in which the breakdown of gas is easier to occur than in air. The result is shown in Fig.B1.

The maximum charge in argon gas for a given size larger than $8 \mu \mathrm{m}$ looks different from that shown before. We can notice also the polarity dependence. The both indicate that the breakdown in argon gas limited the charges. The result of prediction by the Townsend theory for argon gas is also plotted in the solid line. It conforms pretty well to the data for negatively charged particles within $10 \%$ error. (It agrees exactly with the positive data, but this is merely 
accidental, because the prediction is valid only for negative charges.) From this, we may expect the calculation procedure for maximum charge in air for negative polarity is quite reasonable, and therefore, Eq.(B6) is acceptable as a reasonable prediction. Although Eq.(B6) is for negatively charged particles in air at normal room conditions, it may be usable also for positively charged particles because the difference due to the polarity is not very appreciable as shown in Fig.B1.

When the droplets become smaller than $8 \mu \mathrm{m}$, the breakdown limit exceeds the Rayleigh limit, and then the maximum charge in argon gas follows the limiting line in air and $\mathrm{SF}_{6}$ plotted in the bold broken line, which was shown also in Figs.14 and 15.

\section{Notation}

A displacement amplitude of particle oscillation, $\mathrm{m}$

$A_{\mathrm{x}}, A_{\mathrm{z}} \quad$ Amplitudes of $x$ and $z$ components of electric field in the quadrupole electrode assembly $\left(A_{\mathrm{z}}=-A_{\mathrm{x}}\right), \mathrm{V} / \mathrm{m}$

$a, b \quad$ dummy variable or constants

B proportionality constant in Eq.(6), A

$d \quad$ gap size of parallel plate electrode appearing in Paschen curve, $\mathrm{cm}$

$D_{\mathrm{p}} \quad=2 r_{\mathrm{p}}$, particle diameter, $\mathrm{m}$

E $\quad$ electric field strength, $\mathrm{V} / \mathrm{m}$

$E_{\mathrm{x}}, E_{\mathrm{z}} \quad x$ and $z$ components of electric field in the quadrupole electrode assembly, $\mathrm{V} / \mathrm{m}$

$E_{0} \quad$ norm (or absolute value) of the electric field for driving charged particle, $\mathrm{V} / \mathrm{m}$ 
$g \quad$ acceleration constant due to gravity, $\mathrm{kg} \mathrm{m} / \mathrm{s}^{2}$

$h(n) \quad$ relative strength of line spectrum for $\mathrm{n}$-th harmonic, dimensionless

$i_{p} \quad$ ac component of beat signal, A

$J_{\mathrm{n}}(z) \quad$ Bessel function of the first kind of order n, dimensionless

$k=2 \pi / \delta$, wave number of laser interference fringes, $1 / \mathrm{m}$

m $\quad$ particle mass, $\mathrm{kg}$

$n \quad$ order of harmonics, dimensionless

$n_{\mathrm{e}} \quad$ number of electrons bred by a single electron starting from cathode, dimensionless

$p \quad$ ambient pressure, $\mathrm{Pa}$

$p_{20} \quad$ reduced pressure, i.e., the pressure at which the density of gas at $20^{\circ} \mathrm{C}$ equals the density of actual gas surrounding the electrode, $\mathrm{Pa}$

$q \quad$ particle charge, Coulomb

$q_{\max } \quad$ maximum charge for spherical particle sustainable in normal air, Coulomb

$Q_{\mathrm{RL}} \quad$ Rayleigh limit, Coulomb

$R \quad$ drag coefficient, $\mathrm{kg} / \mathrm{s}$

$r \quad$ radius coordinate, $\mathrm{m}$

$r_{\mathrm{p}} \quad$ radius of charged particle, $\mathrm{m}$

$r_{0} \quad$ radius coordinate at which the field strength becomes too weak for ionization collision

to occur, $\mathrm{m}$ (The critical field strength is $24.5 \mathrm{kV} / \mathrm{cm}$ in normal air.)

$t \quad$ time, $\mathrm{s}$ 
$V_{\mathrm{bk}} \quad$ breakdown voltage for parallel plate electrode, $\mathrm{V}$

$x, y, z \quad$ rectangular coordinates, $\mathrm{m}$

$x_{0} \quad$ initial position of particle in the fringes, see Eq.(5), m

Z drag force, $\mathrm{N}$

\section{Greek letters}

$\alpha \quad$ ionization coefficient, $1 / \mathrm{cm}$

$\delta \quad$ fringe spacing, $\mu \mathrm{m}$

$\varepsilon \quad$ permittivity of medium gas $\left(\approx 10^{-9} / 36 \pi\right)$, farad $/ \mathrm{m}$

$\phi \quad$ phase lag of particle displacement with respect to applied ac electric field, rad

$\gamma \quad$ secondary electron emission coefficient, dimensionless

$\gamma_{0} \quad \gamma$ for brass in normal air estimated from the Paschen curve, dimensionless

$\lambda \quad=r_{\mathrm{p}} \sqrt{\omega_{\mathrm{p}} / v}$, size parameter, dimensionless

$v \quad$ kinematic viscosity, $\mathrm{m}^{2} / \mathrm{s}$

$\rho_{\mathrm{f}} \quad$ density of fluid, $\mathrm{kg} / \mathrm{m}^{3}$

$\rho_{\mathrm{p}} \quad$ density of particle, $\mathrm{kg} / \mathrm{m}^{3}$

$\sigma \quad$ surface tension, $\mathrm{N} / \mathrm{m}$

$\omega \quad$ angular frequency of electric field for trapping charged particle, $\mathrm{rad} / \mathrm{s}$

$\omega_{\mathrm{p}} \quad$ angular frequency of particle oscillation, $\mathrm{rad} / \mathrm{s}$

$\psi \quad=\phi-\pi / 2$, phase lag of particle velocity with respect to applied electric field, rad 


\section{References}

Abbas, M.A. Latham, J., (1967). The stability of evaporating charged drops. Journal of Fluid Mechanics 30, .663-670

Ashkin, A., Dziedzic J.M., (1981). Observation of optical resonances of dielectric spheres by light scattering. Applied Optics 20, 1803-1814

Bartholdi, M., Salzman, G.C.,.Hiebert, R.D.,.Kerker, M., (1980). Differential light scattering photometer for rapid analysis of single particle in flow. Applied Optics 19, 1573-1584

Bhanti, D., Ray, A.K., (1998). In situ measurement of photochemical reactions in microdroplets. Journal of Aerosol Science 30, 279-287

Blackman, R.B., Tukey, J.W., (1958). The measurement of power spectra, Dover Publications Inc., New York, p.14.

Chandezon, F., Tomita, S., Cormier, D., Grübling, P., Guet, C., Lebius, H., Pesnelle, A., Huber, B.A., (2001). Rayleigh instabilities in multiply charged sodium clusters. Physical Review Letters 87, 153402-1-4 
Crowley, J.M., (1991). Fundamentals of Applied Electronics, Krieger Publishing Co., Malabar Florida, p.28

Davis, E.J., Bridges, M,A., (1994). The Rayleigh limit of charge revisited: light scattering from exploding droplets. Journal of Aerosol Science 25, 1179-1199

Duft, D., Achtzehn, T., Müller, R., Huber, B.A., Leisner, T., (2003). Rayleigh jets from levitated microdroplet. Nature 421, 128 (09 January)

Duft ,D., Lebius, H., Huber, B.A., Guet, C., Leisner, T., (2002) Shape oscillation and stability of charged microdroplets, Physical Review Letters 19, 084503.1-084503.4

Dutton, J., (1975). Electron swarm data, Journal of Physical Chemistry Reference Data (Table 7.9) 4, 730

Elghazaly, H.A., Castle, G.S.P., (1987). The charge limit of liquid droplets due to electron avalanches and surface disruption, Electrostatics 1987, IOP Publishing Ltd, Bristol, pp.121-126.

Engel, A., (1965). Ionized Gases (Chapter 7), Clarendon Press, Oxford, pp.171-216. 
Farmer, W.M., (1974) Observation of large particles with a laser interferometer, Applied Optics 13, 610-622

Farmer, A., (1972) Measurement of particle size, number density, and velocity using a laser interferometer, Applied Optics 11, 2603-2612

Fernández de la Mora, J., (1996). On the outcome of the Coulombic fission of a charged isolated drop. Journal of Colloid and Interface Science 178, 209-218

Harper, W.R., (1967). Contact and Frictional Electrification, Oxford University Press, London, pp.13-15

Hartmann, G., (1984). Theoretical evaluation of Peek’s law. IEEE on Industry Applications 20, $1647-1651$

Hendricks, C.D., Schneider, J.M., (1963). Stability of a conducting droplet under the influence of surface tension and electrostatic forces. American Journal of Physics 31, 450-453

Hinze, J.O., (1959). Turbulence, McGraw-Hill, New York, p.354 
Masuda, S., Washizu, M., Sekiguchi, T., (1980) Electrostatic method of pellet handling, IAS

Conference Record 1980 (IEEE), 1005-1010

Mazumder, M.K., Kirsch, K.J., (1977). Single particle aerodynamic relaxation time analyzer.

Review of Scientific Instruments 48, 622-624

Meek, J.K., (1940). A theory of spark discharge. Physical.Review57, 722-728

Nakajima, Y., Sato, T., (2003). Electrostatic collection of submicron particles with the aid of electrostatic agglomeration promoted by particle vibration. Powder Technology 135-136, 266-284

Nakajima, Y., Sato, T., (1999a). Estimation of maximum charge sustainable for a spherical particle in normal air (in Japanese). Journal of Institution of Electrostattics, Japan 23,.81-87

Nakajima, Y., Sato, T., (1999b). On the estimation of maximum charge sustainable for a positively charged spherical particle by the Meek's theory (in Japanese). Journal of Institution of Electrostattics, Japan 23,.325-326 
Pedersen, A., (1967). Analysis of spark breakdown characteristics for sphere gaps. IEEE Transactions on Power Apparatus and Systems 86, 975-978

Pedersen, A., (1989). On the electric breakdown of gaseous dielectrics. IEEE Transactions on Electrical Insulation 24, 721-739

Pipes, L.A., Harvill, L.R. (1970). Applied mathematics for engineers and physicists (3rd Ed.), International Student Edition, McGraw-Hill Kogakusha, Tokyo, p.798

Renninger, R.G., Mazumder, M.K., Testerman, M.K., (1981). Particle sizing by electrical single particle aerodynamic relaxation time analyzer. Review of Scientific Instruments 52, 242-246

Rayleigh, Lord J.W.S., (1882). On the equilibrium of liquid conducting mass charged with electricity. Philosophical Magazine 14 (5th Series), 184-186

Richardson, C.B., Pigg, A.L., Hightower, R.L., (1989). On the stability limit of charged droplet. Proceeding of Royal Society of London A 422, 319-328

Roberds, D.W., (1977). Particle sizing using laser interferometry. Applied Optics 16, 1861-1868 
Sato, T., Nakajima, Y.,(1990). Method for simultaneous measurement of size and charge of fine particles.(in Japanese), Transaction of Institution of Electrical Engineering Japan 110-A, 473-482

Schumann, W.O. ( 1923). Elektrische Durchbruch-feldsterke von Gasen, Springler-Verlag, Berlin, pp.170-177

Schwell, M., Baumgärtel, H., Weidinger, I., Kämer, B., Vortisch, H., Wöste, L., Leisner, T., Rühl, E., (2000). Uptake dynamics and diffusion of $\mathrm{HCl}$ in sulfuric acid solution measured in single levitated microdroplets. Journal of Physical Chemistry A 104, 6726-6732

Shulman, M.L., Charlson, R.J., Davis, E.J., (1997). The effect of atmospheric organics on aqueous droplet evaporation. Journal of Aerosol Science 28, 737-751

Taflin, D,C., Zhang, S.H., Allen, T., Davis, E.J., (1988). Measurement of droplet interfacial phenomena by light-scattering techniques. AIChE Journal 34, 1310-1320

Taflin, D.C., Ward, T.L., Davis, E.J. (1989). Electrified droplet fission and the Rayleigh limit. Langmure, 5, 376-384

Ward, T.L., Zhang, S.H.,Allen, T., Davis, E.J., (1987). Photochemical polymerization of 
acrylamide aerosol particles. Journal of.Colloid and Interface Science 118, 343-355

Wells, P.V., Gerke, R.H., (1919). An oscillation method for measuring the size of ultramicroscopic particles. Journal of American Chemical Society 41,312-329

Widman, J.F., Heusmann, C.M., Davis, E.J., (1998). The effect of polymeric additive on the evaporation of organic aerocolloidal droplets. Colloid and Polymer Science 276, 197-205

Wind, G., (1974). ELECTRA (CIGRÉ), Paschen curve for air. No.32, 70-74

Wuerker, R.F., Shelton, H., Langmuir, R.V., (1959). Electrodynamic containment of charged particles. Journal of Applied Physics 30, 342-349

Zhu, J., Zheng, F., Laucke, M.L., Davis, E.J., (2002). Mass transfer from an oscillating microsphere. Journal of Colloid and Interface Science 249, 351-358 


\section{Figure Captions}

Fig.1 Quadrupole electrode assembly for trapping charged particle (For negatively charged particles, polarity of diodes should be reversed.)

Fig.2 Equipotential and electric force lines in the quadrupole electrode assembly

Fig.3 Absolute value of electric field vector in the center of the quadrupole electrodes

Fig.4 Example of Doppler signal from a single particle with an amplitude, $A$, larger than the fringe spacing, $\delta$. ( $A \approx 7.7 \delta=73 \mu \mathrm{m}$.) The phase lag $\phi$ corresponds to the time interval between the reversal points of the drive signal and the particle oscillation.

Fig.5 Example of beat signal for an amplitude smaller than the fringe size

Fig.6 Amplitude spectrum of beat signal shown in Fig.5

Fig.7 Relative intensity of each harmonic component in amplitude spectrum

Fig.8 Schematic diagram of the measuring system

Fig.9 Example of accuracy check

Fig.10 Sensitivity of size to phase error relative to optimum condition

Fig.11 Time variations in size and charge of evaporating DBP droplet

Fig.12 Time variations in size and charge of evaporating Pentadecane droplet

Fig.13 Time variations in size and charge of evaporating glycerol droplet

Fig.14 Maximum charge vs. droplet diameter for glycerol in air and $\mathrm{SF}_{6}$ (positive and negative)

Fig. 15 Predicted maximum charge of spherical particle in normal atmospheric conditions

Fig. A1 Map of angle corrections required for $A_{3}{ }^{*}$ and $S_{3}{ }^{*}$ when $\phi$ and $k x_{0}$ are given

Fig. B1 Electric breakdown observed in Argon gas 


\begin{tabular}{|c|c|c|c|c|c|}
\hline mode & $\phi^{*}$ & (a) & (b) & (c) & (d) \\
\hline 0th & undefined & $\begin{array}{c}\phi: \text { undifined } \\
k x_{0}=k x_{0}^{*}\end{array}$ & & & \\
\hline $\begin{array}{c}\text { funda } \\
\text { m. }\end{array}$ & $\begin{array}{c}-\pi / 2 \square 0 \\
\pi / 2 \square \pi\end{array}$ & $\begin{array}{c}\phi=\phi^{*} \\
k x_{0}=k x_{0}^{*} \\
\phi=\phi^{*} \\
k x_{0}=k x_{0}^{*}\end{array}$ & $\begin{aligned} \phi & =\phi^{*}+\pi \\
k x_{0} & =k x_{0}^{*} \pm \pi \\
\phi & =\phi^{*}-\pi \\
k x_{0} & =k x_{0}^{*} \pm \pi\end{aligned}$ & & \\
\hline second & $\begin{array}{l}-\pi / 2 \square 0 \\
0 \square \pi / 2\end{array}$ & $\begin{array}{c}\phi=\phi^{*} \\
k x_{0}=k x_{0}^{*} \\
\phi=\phi^{*}-\pi / 2 \\
k x_{0}=k x_{0}^{*} \pm \pi\end{array}$ & $\begin{array}{c}\phi=\phi^{*}+\pi \\
k x_{0}=k x_{0}^{*} \\
\phi=\phi^{*}+\pi / 2 \\
k x_{0}=k x_{0}^{*} \pm \pi\end{array}$ & & \\
\hline third & $\begin{array}{c}-\pi / 3 \square-\pi / 6 \\
-\pi / 6 \square 0 \\
0 \square \pi / 6 \\
\pi / 6 \square \pi / 3\end{array}$ & $\begin{array}{c}\phi=\phi^{*} \\
k x_{0}=k x_{0}^{*} \\
\phi=\phi^{*}-\pi / 3 \\
k x_{0}=k x_{0}^{*} \pm \pi \\
\phi=\phi^{*}-\pi / 3 \\
k x_{0}=k x_{0}^{*} \pm \pi \\
\phi=\phi^{*}-2 \pi / 3 \\
k x_{0}=k x_{0}^{*}\end{array}$ & $\begin{array}{c}\phi=\phi^{*}+\pi \\
k x_{0}=k x_{0}^{*} \pm \pi \\
\phi=\phi^{*} \\
k x_{0}=k x_{0}^{*} \\
\phi=\phi^{*}+2 \pi / 3 \\
k x_{0}=k x_{0}^{*} \\
\phi=\phi^{*}-\pi / 3 \\
k x_{0}=k x_{0}^{*} \pm \pi\end{array}$ & $\begin{array}{c}\phi=\phi^{*}+2 \pi / 3 \\
k x_{0}=k x_{0}^{*} \\
\\
\phi=\phi^{*}+\pi / 3 \\
k x_{0}=k x_{0}^{*} \pm \pi\end{array}$ & $\begin{array}{c}\phi=\phi^{*}+\pi \\
k x_{0}=k x_{0}^{*} \pm \pi \\
\phi=\phi^{*}+2 \pi / 3 \\
k x_{0}=k x_{0}^{*}\end{array}$ \\
\hline fourth & $\begin{array}{c}-\pi / 4 \square 0 \\
0 \square \pi / 4\end{array}$ & $\begin{array}{c}\phi=\phi^{*}-\pi / 4 \\
k x_{0}=k x_{0}^{*} \pm \pi \\
\phi=\phi^{*}-\pi / 2 \\
k x_{0}=k x_{0}^{*}\end{array}$ & $\begin{array}{c}\phi=\phi^{*} \\
k x_{0}=k x_{0}^{*} \\
\phi=\phi^{*}-\pi / 4 \\
k x_{0}=k x_{0}^{*} \pm \pi\end{array}$ & $\begin{array}{c}\phi=\phi^{*}+3 \pi / 4 \\
k x_{0}=k x_{0}^{*} \pm \pi \\
\phi=\phi^{*}+\pi / 2 \\
k x_{0}=k x_{0}^{*}\end{array}$ & $\begin{array}{c}\phi=\phi^{*}+\pi \\
k x_{0}=k x_{0}^{*} \\
\phi=\phi^{*}+3 \pi / 4 \\
k x_{0}=k x_{0}^{*} \pm \pi\end{array}$ \\
\hline
\end{tabular}

Table 1 Possible solutions for phase lag, $\phi$, and initial phase, $k x_{0}$, for several harmonic modes ( $\phi^{*}$ and $k x_{0} *$ are directly determined by DFT for $\phi$ and $k x_{0}$, but they may be folded in the range between $-\pi$ to $\pi$. ) 


\section{Figure Captions}

Fig.1 Quadrupole electrode assembly for trapping charged particle (For negatively charged particles, polarity of diodes should be reversed.)

Fig.2 Equipotential and electric force lines in the quadrupole electrode assembly

Fig.3 Absolute value of electric field vector in the center of the quadrupole electrodes

Fig.4 Example of Doppler signal from a single particle with an amplitude, $A$, larger than the fringe spacing, $\delta$. $(A \approx 7.7 \delta=73 \mu \mathrm{m}$.) The phase lag $\phi$ corresponds to the time interval between the reversal points of the drive signal and the particle oscillation.

Fig.5 Example of beat signal for an amplitude smaller than the fringe size

Fig.6 Amplitude spectrum of beat signal shown in Fig.5

Fig.7 Relative intensity of each harmonic component in amplitude spectrum

Fig.8 Schematic diagram of the measuring system

Fig.9 Example of accuracy check

Fig.10 Sensitivity of size to phase error relative to optimum condition

Fig.11 Time variations in size and charge of evaporating DBP droplet

Fig.12 Time variations in size and charge of evaporating Pentadecane droplet

Fig.13 Time variations in size and charge of evaporating glycerol droplet

Fig.14 Maximum charge vs. droplet diameter for glycerol in air and $\mathrm{SF}_{6}$ (positive and negative)

Fig. 15 Predicted maximum charge of spherical particle in normal atmospheric conditions

Fig. A1 Map of angle corrections required for $A_{3}{ }^{*}$ and $S_{3}{ }^{*}$ when $\phi$ and $k x_{0}$ are given

Fig. B1 Electric breakdown observed in Argon gas 


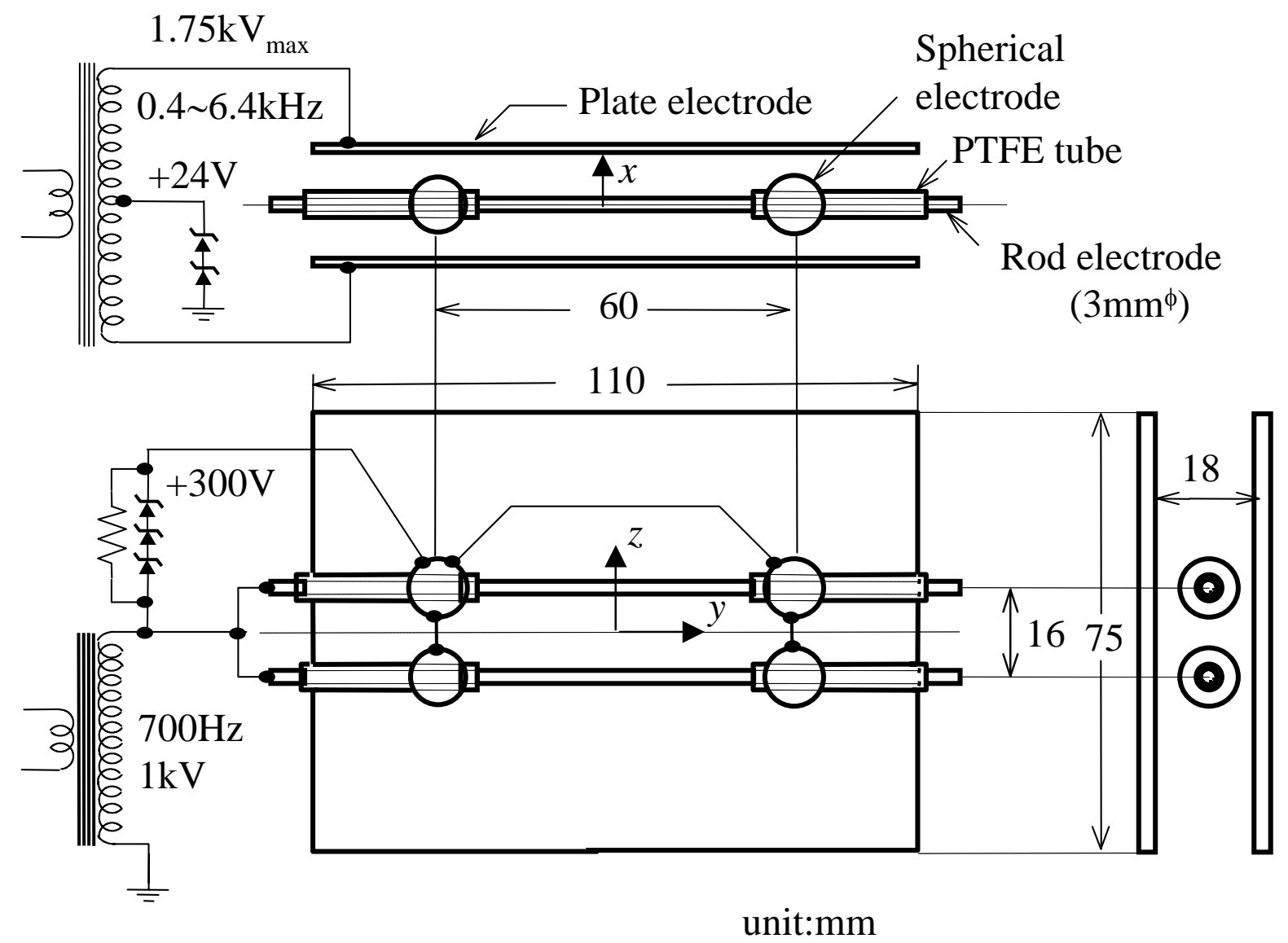

Fig.1 Quadrupole electrode assembly for trapping charged particle

(For negatively charged particles, polarity of diodes should be reversed.) 


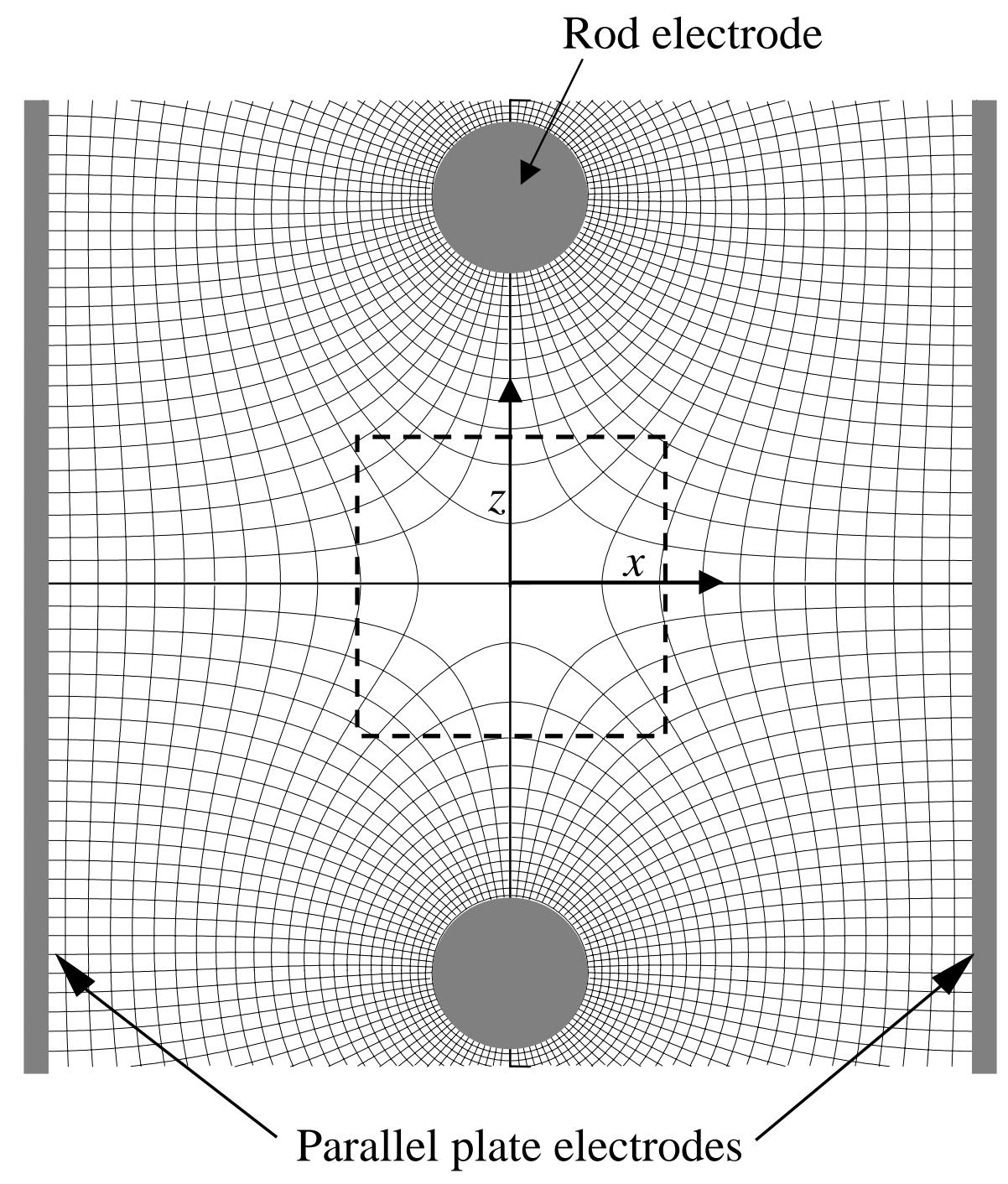

Fig.2 Equipotential and electric force lines in the quadrupole electrode assembly 


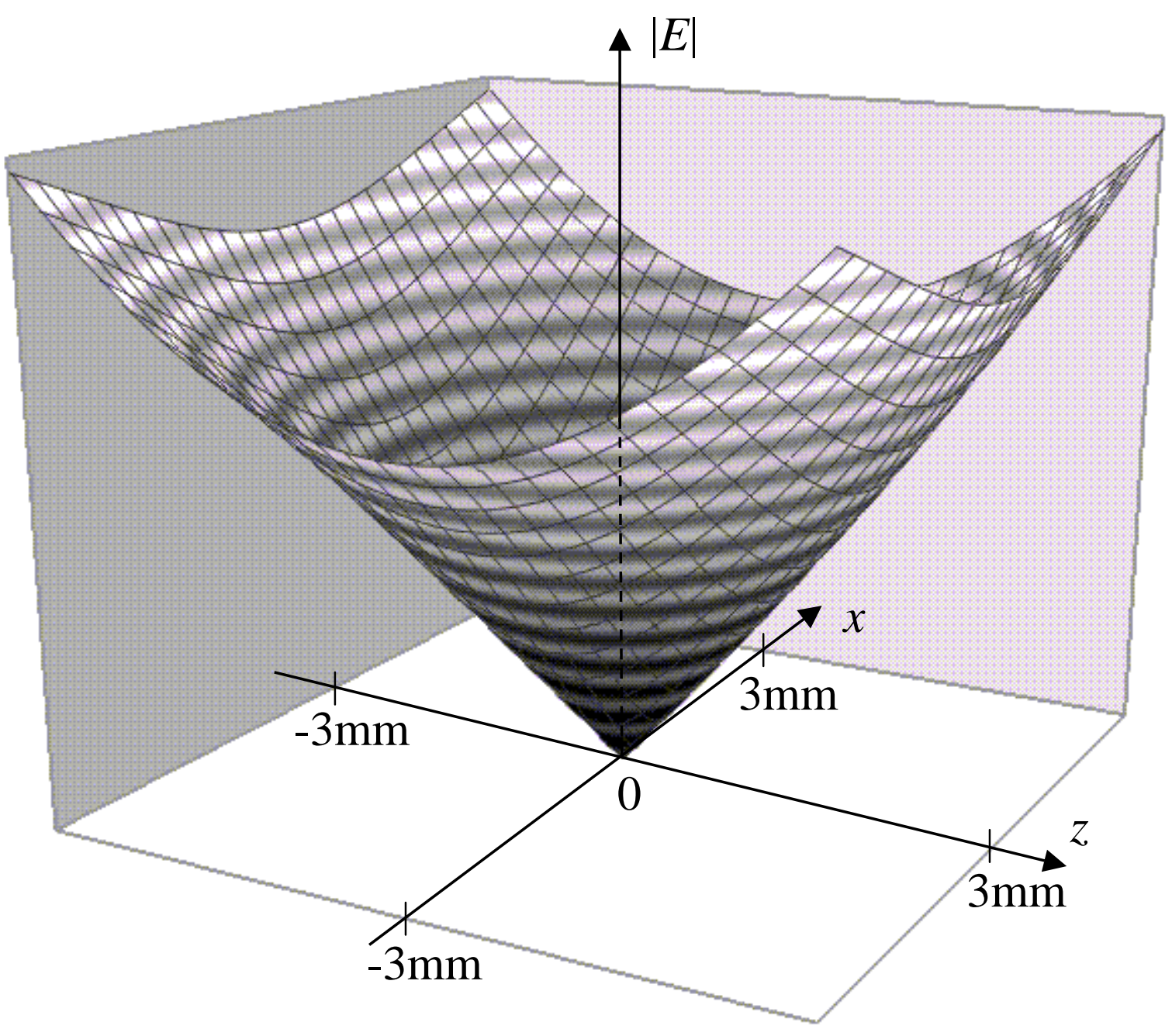

Fig.3 Absolute value of electric field vector in the center of the quadrupole electrodes 


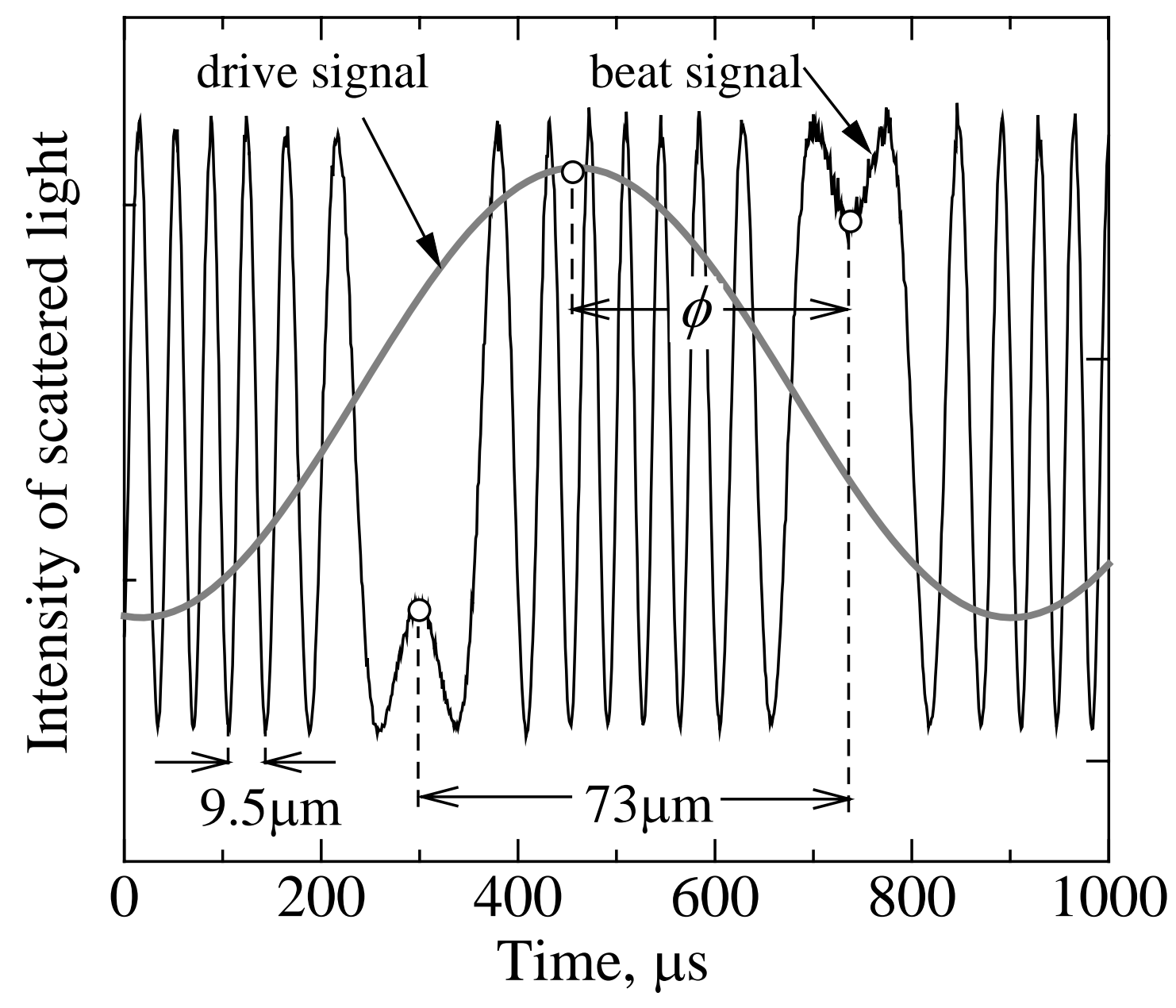

Fig.4 Example of Doppler signal from a single particle with an amplitude, $A$, larger than the fringe spacing, $\delta$. $(A \approx 7.7 \delta=73 \mu \mathrm{m}$.) The phase lag $\phi$ corresponds to the time interval between the reversal points of the drive signal and the particle oscillation 


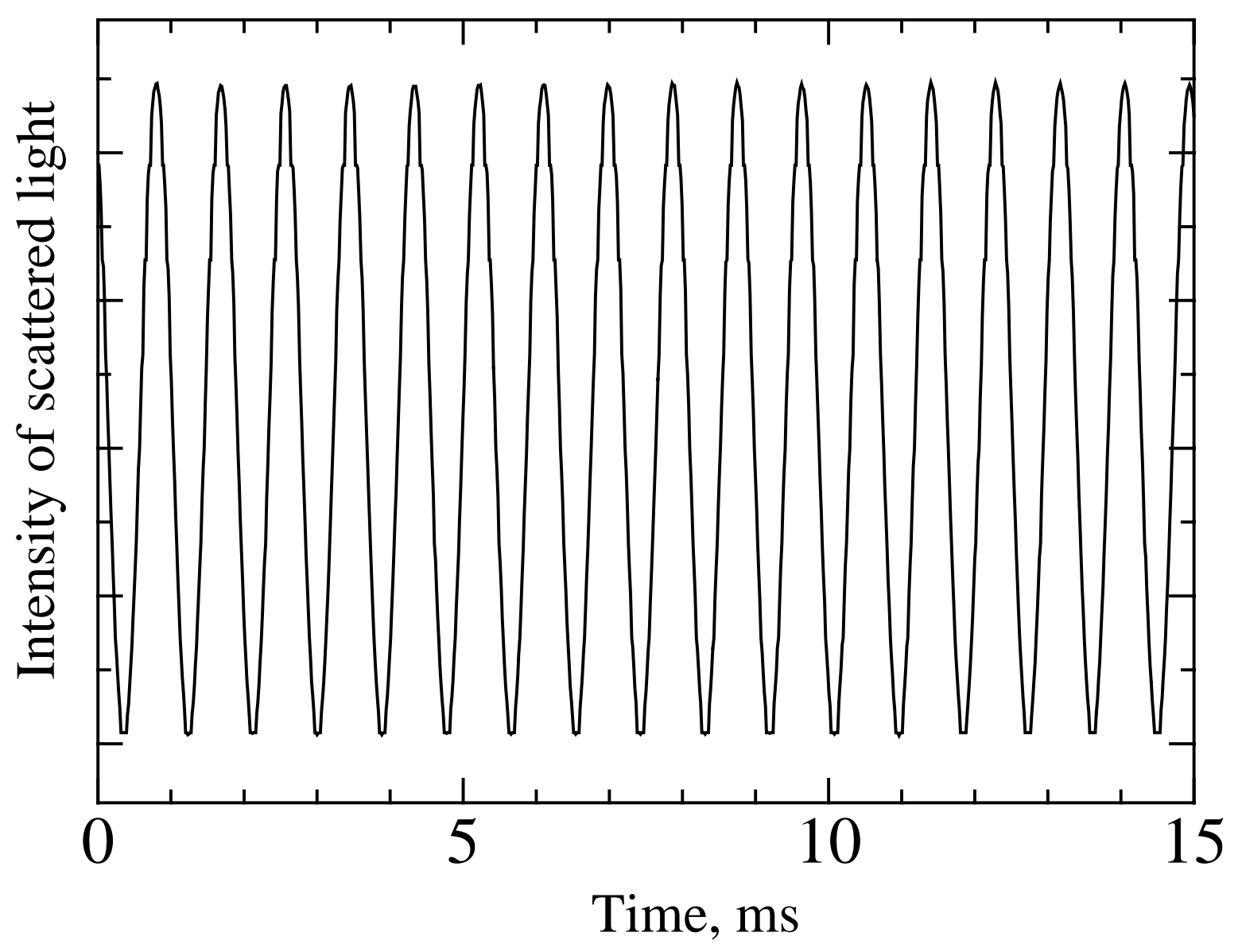

Fig.5 Example of beat signal for an amplitude smaller than the fringe size 


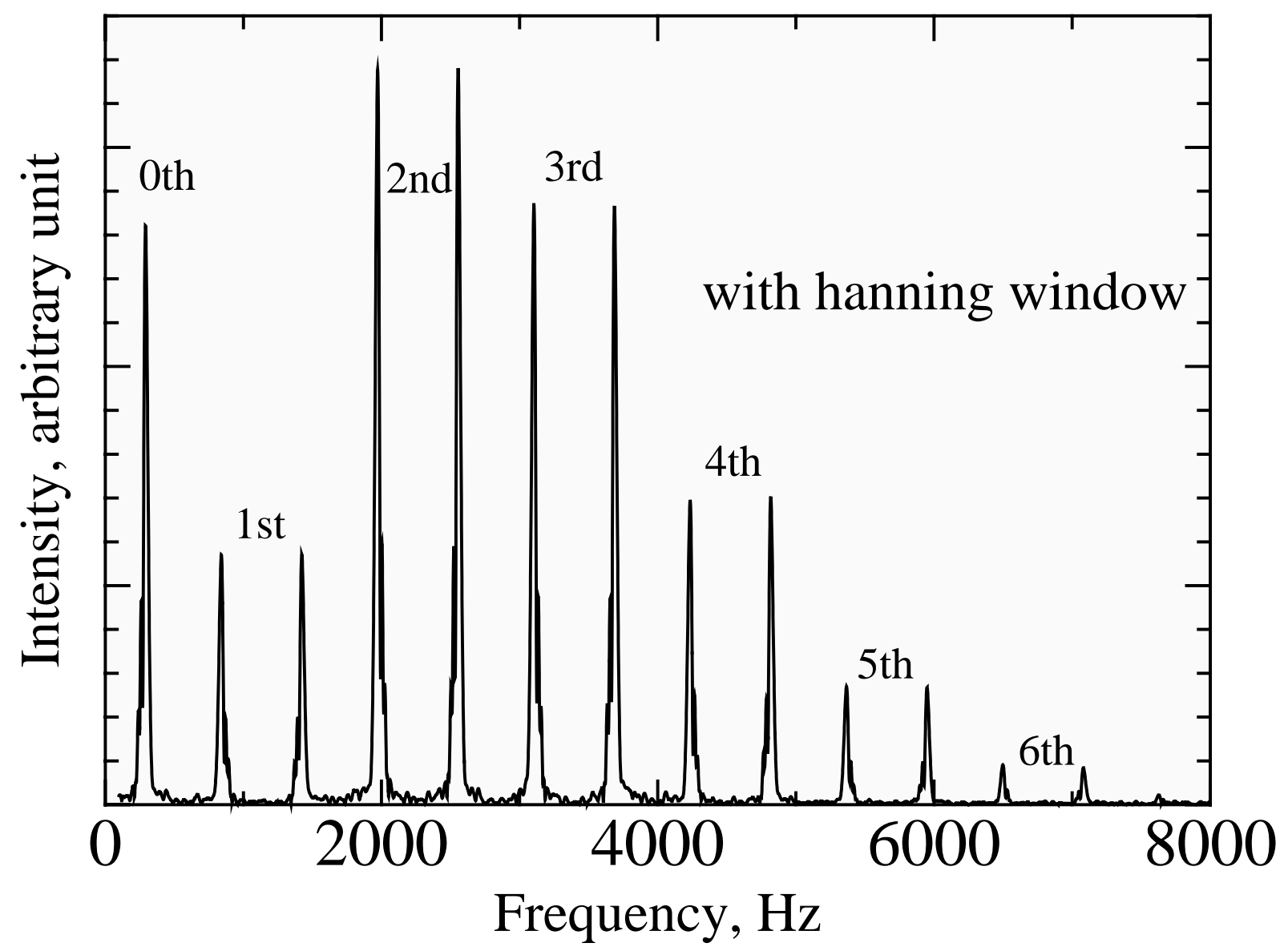

Fig.6 Amplitude spectrum of beat signal shown in Fig.5 


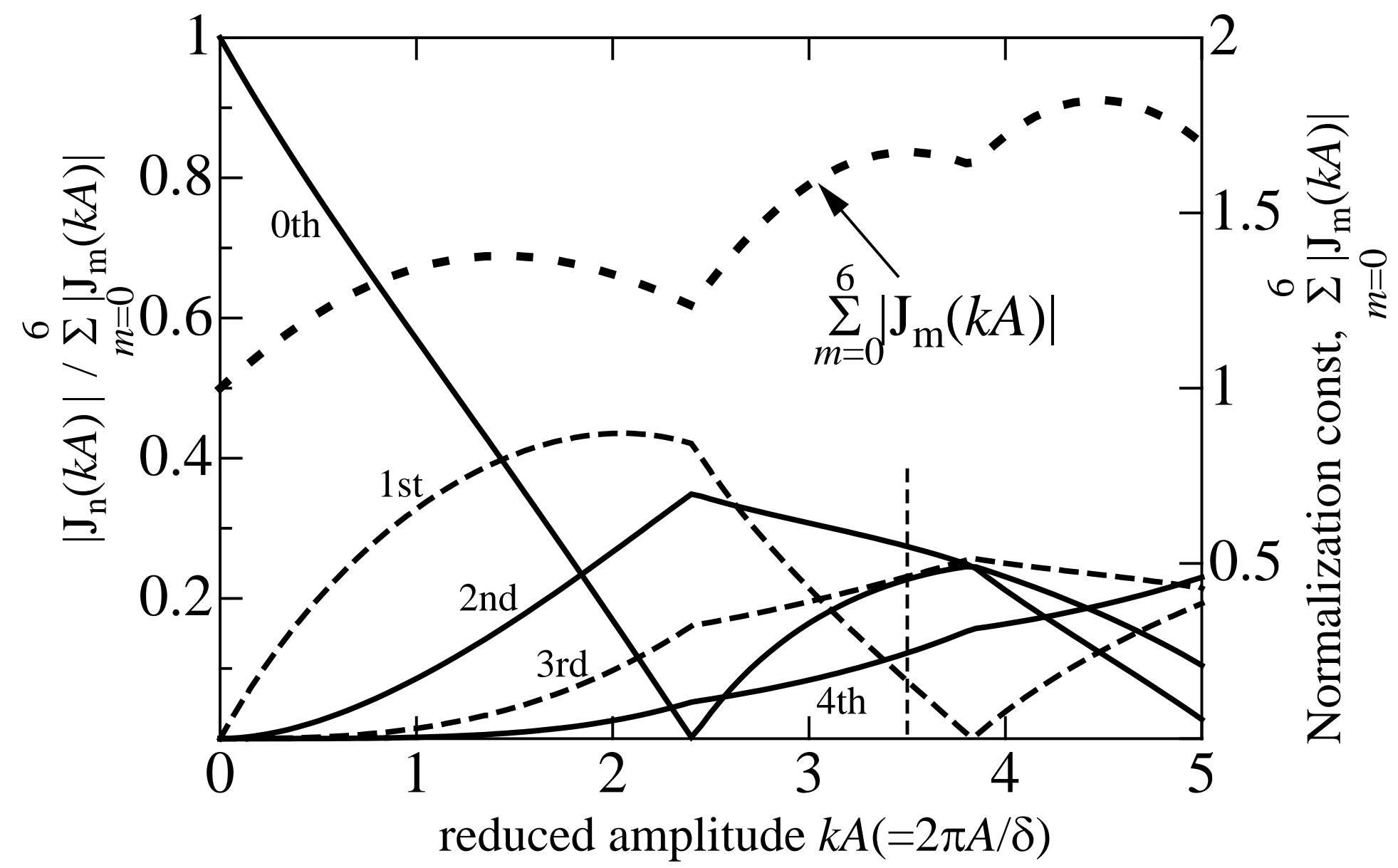

Fig.7 Relative intensity of each harmonic component in amplitude spectrum 


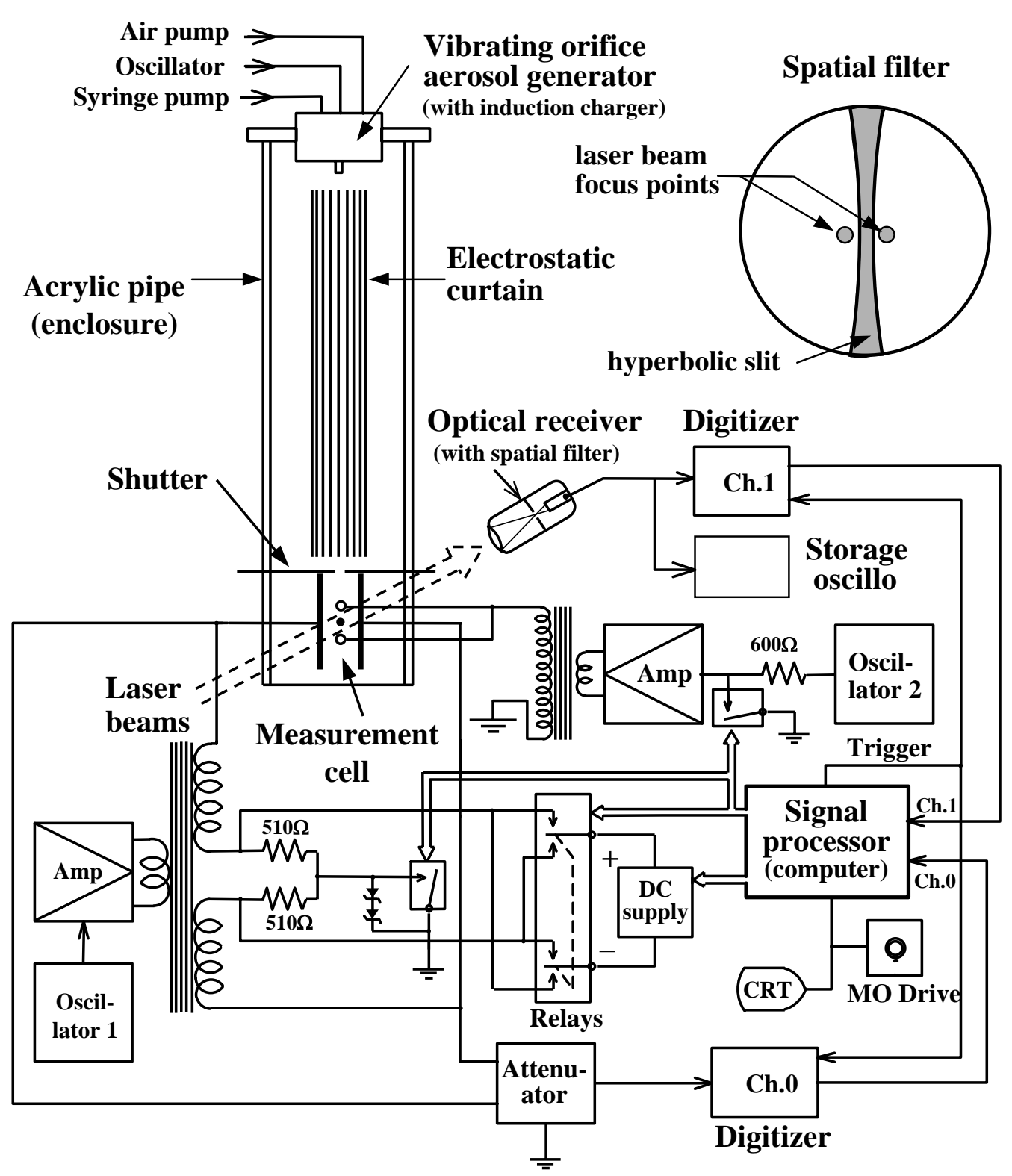

Fig.8 Schematic diagram of the measuring system 


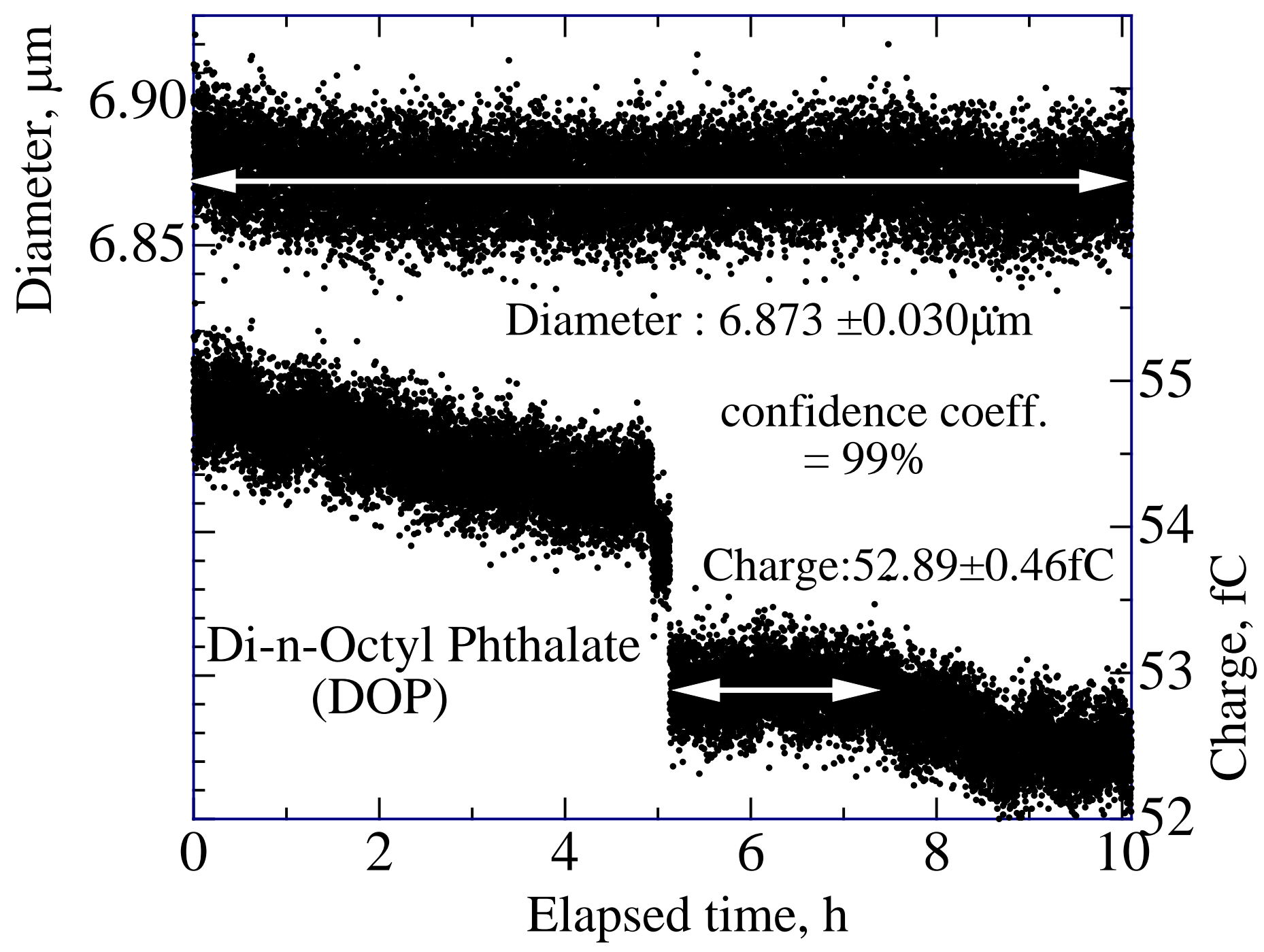

Fig.9 Example of accuracy check 


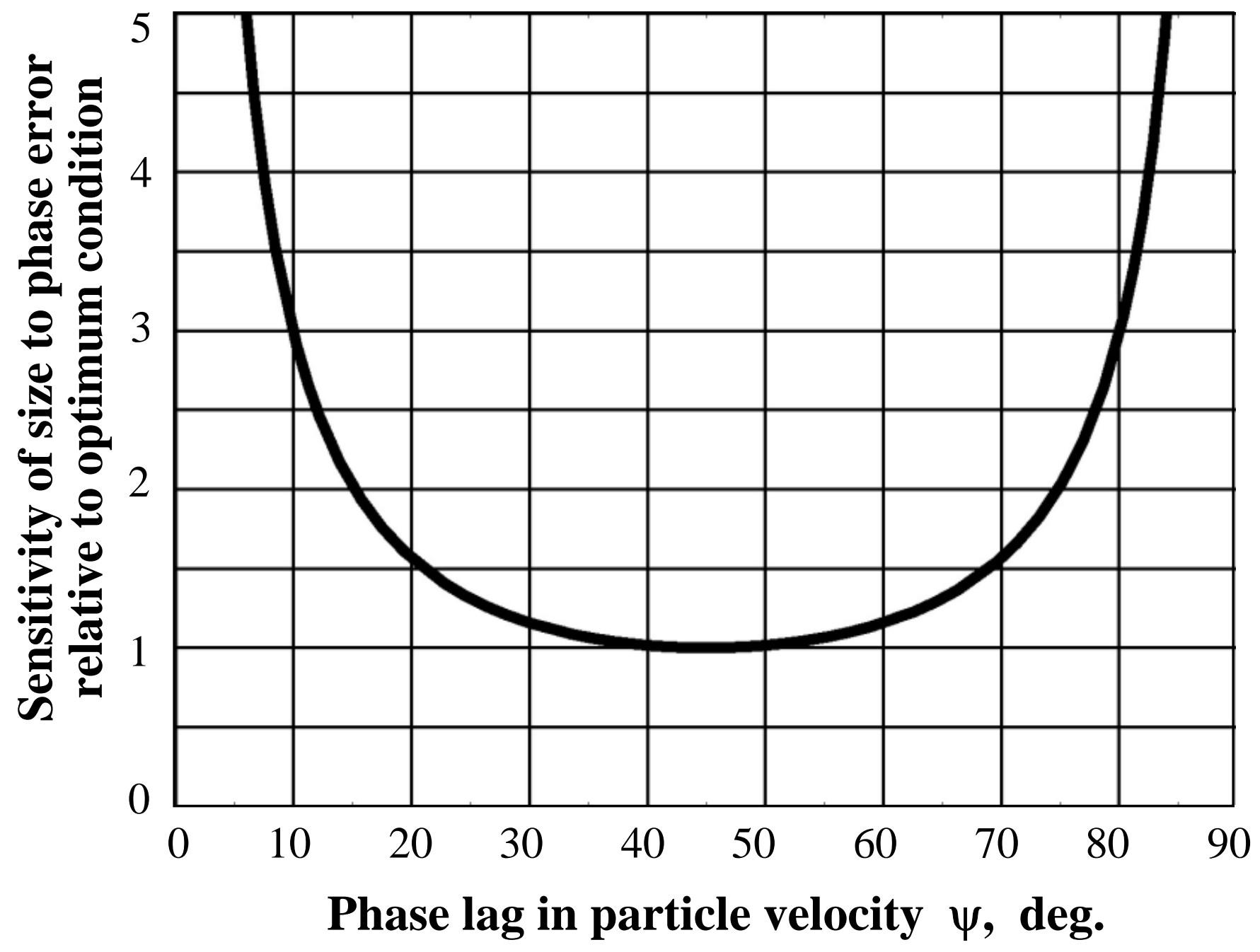

Fig.10 Sensitivity of size to phase error relative to optimum condition 


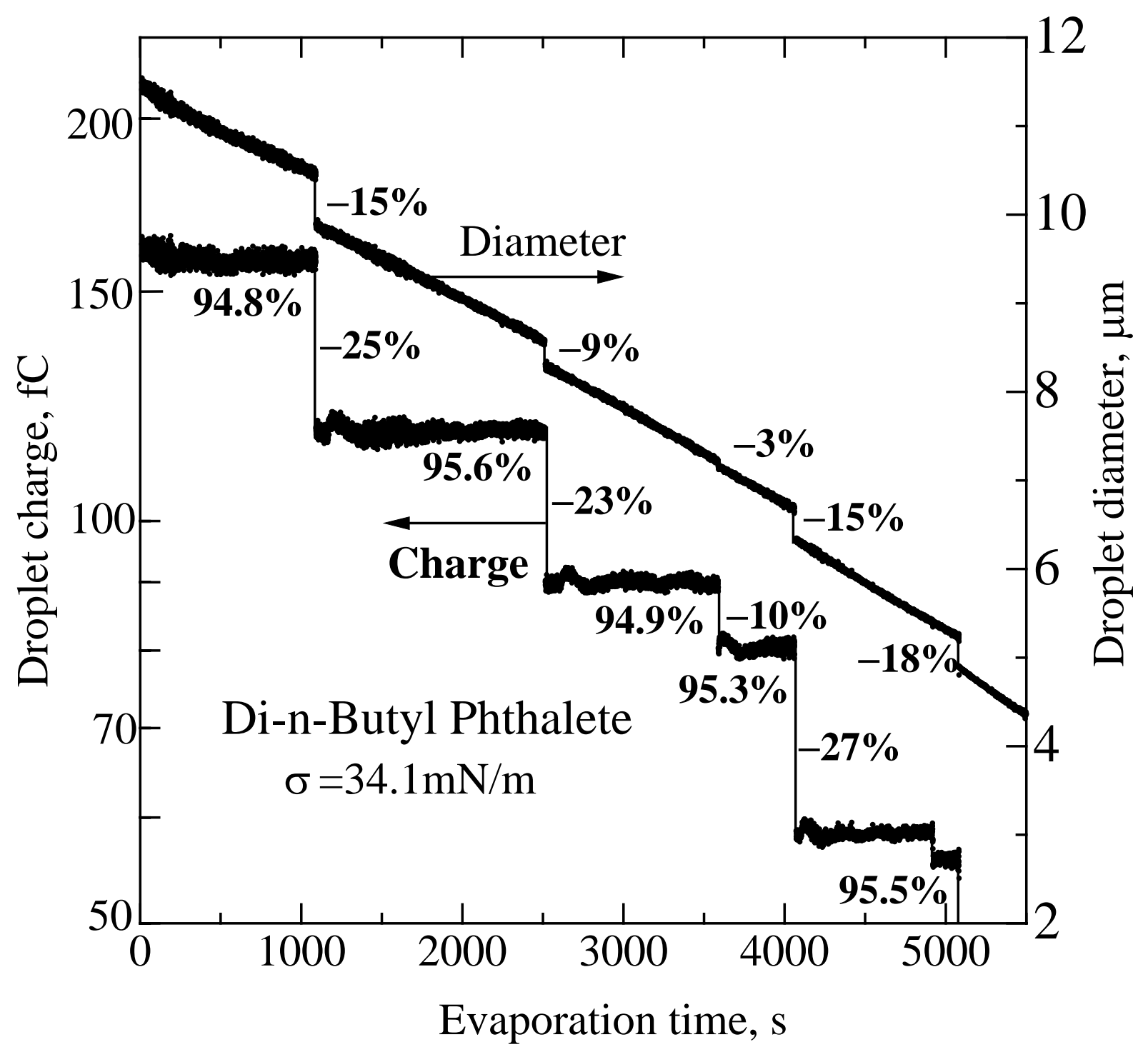

Fig.11 Time variations in size and charge of evaporating DBP droplet 


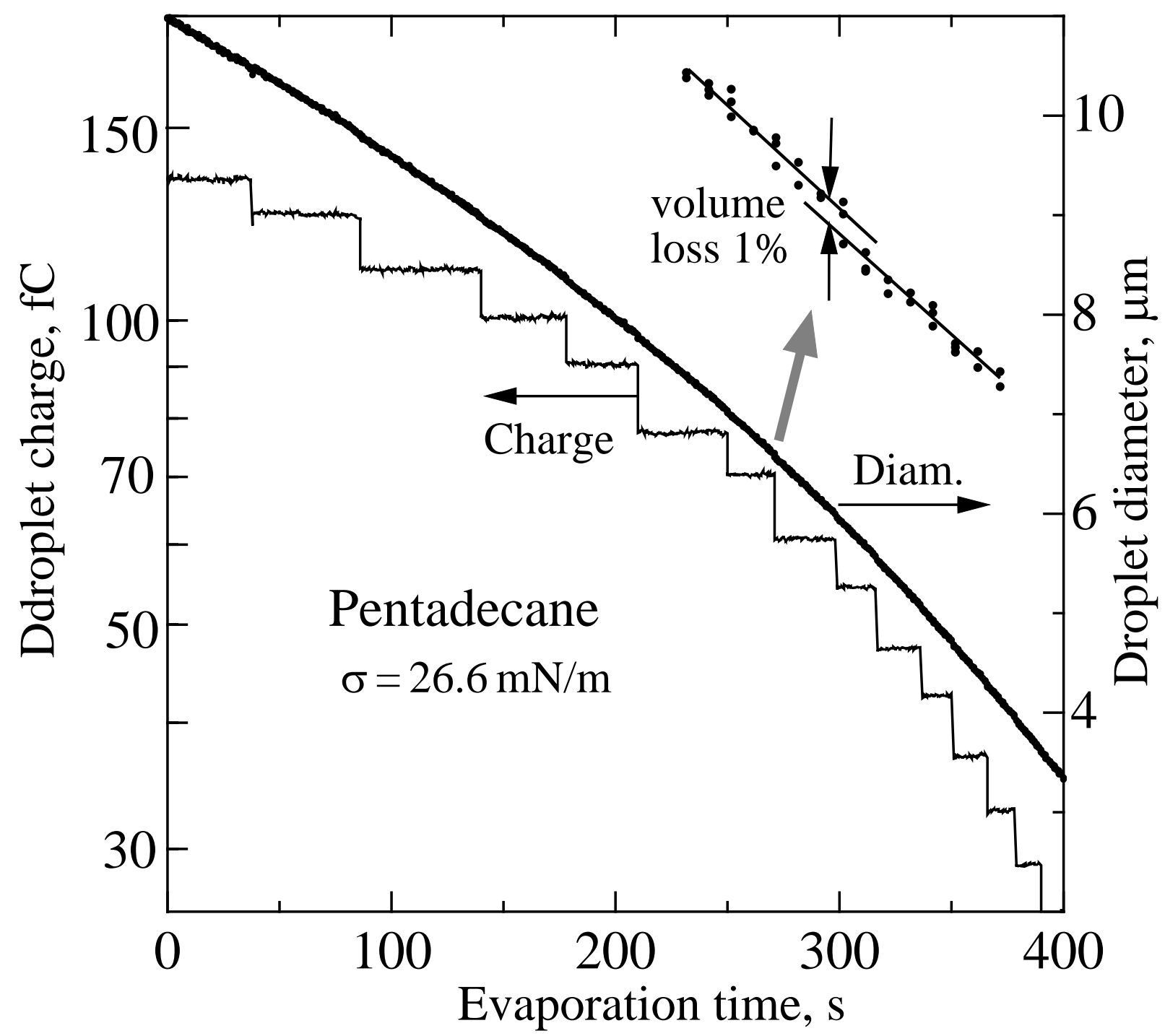

Fig.12 Time variations in size and charge of evaporating Pentadecane droplet 


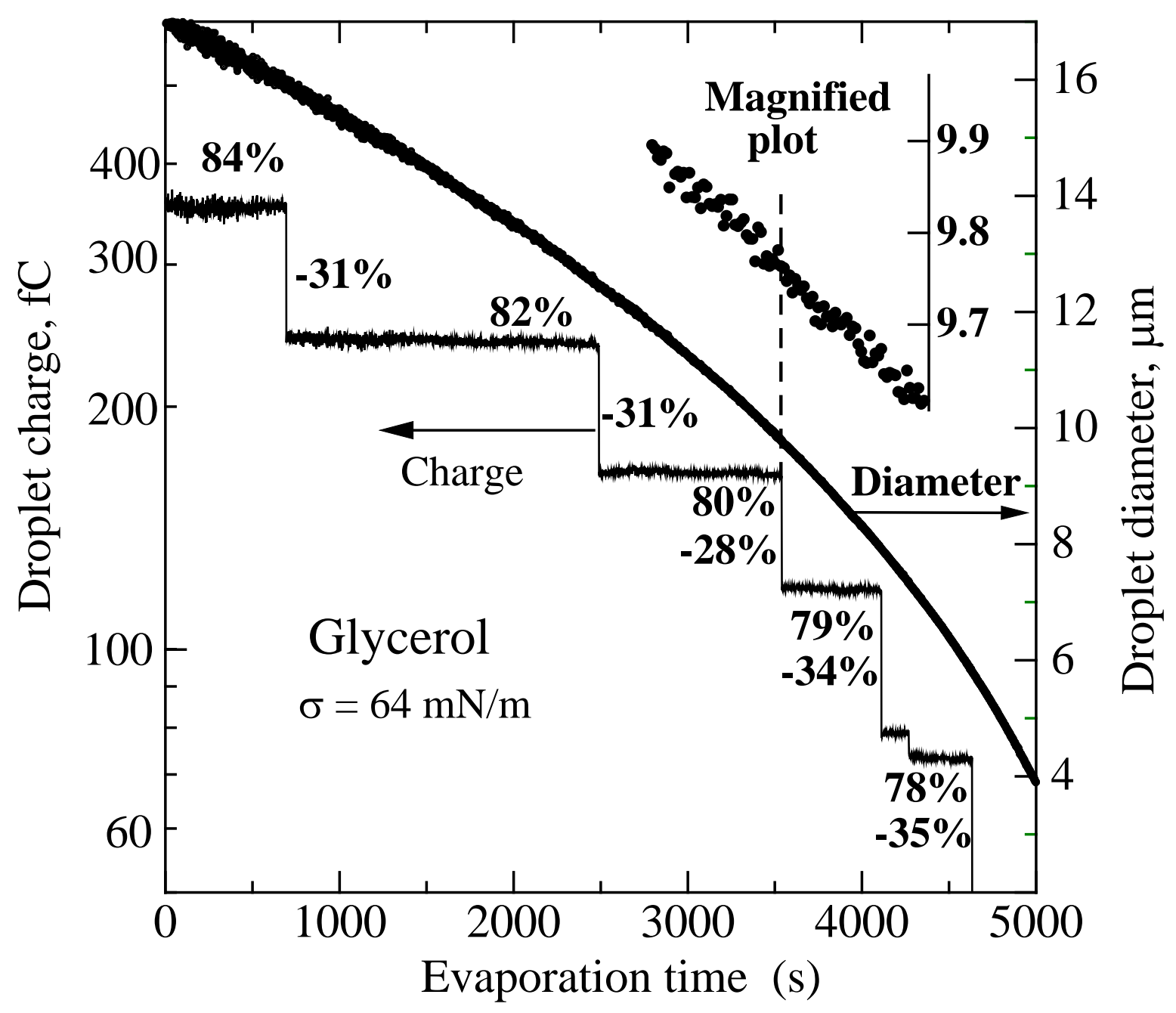

Fig.13 Time variations in size and charge of evaporating glycerol droplet 


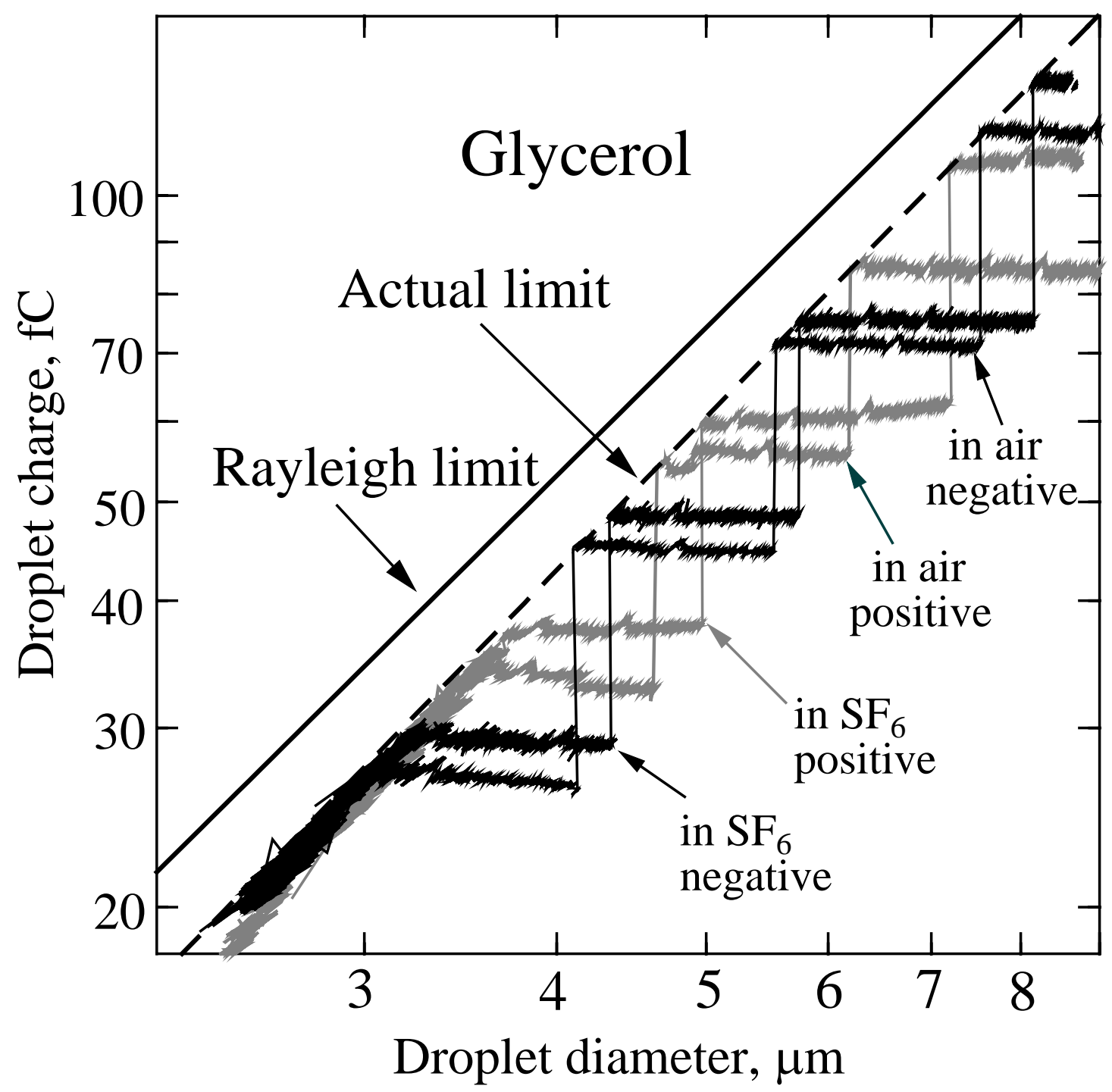

Fig.14 Maximum charge vs. droplet diameter for glycerol in air and $\mathrm{SF}_{6}$ (positive and negative) 


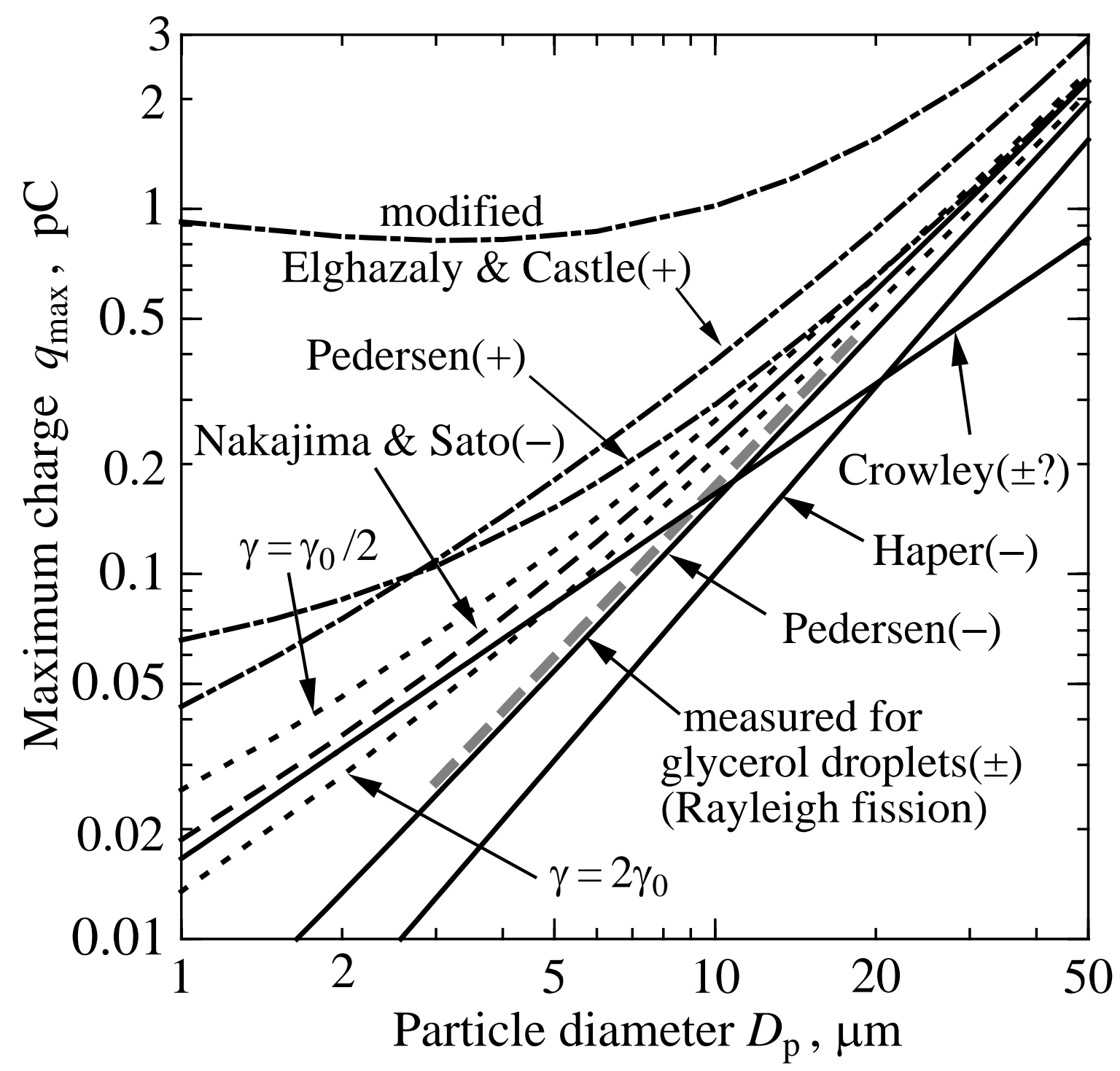

Fig. 15 Predicted maximum charge of spherical particle in normal atmospheric conditions 


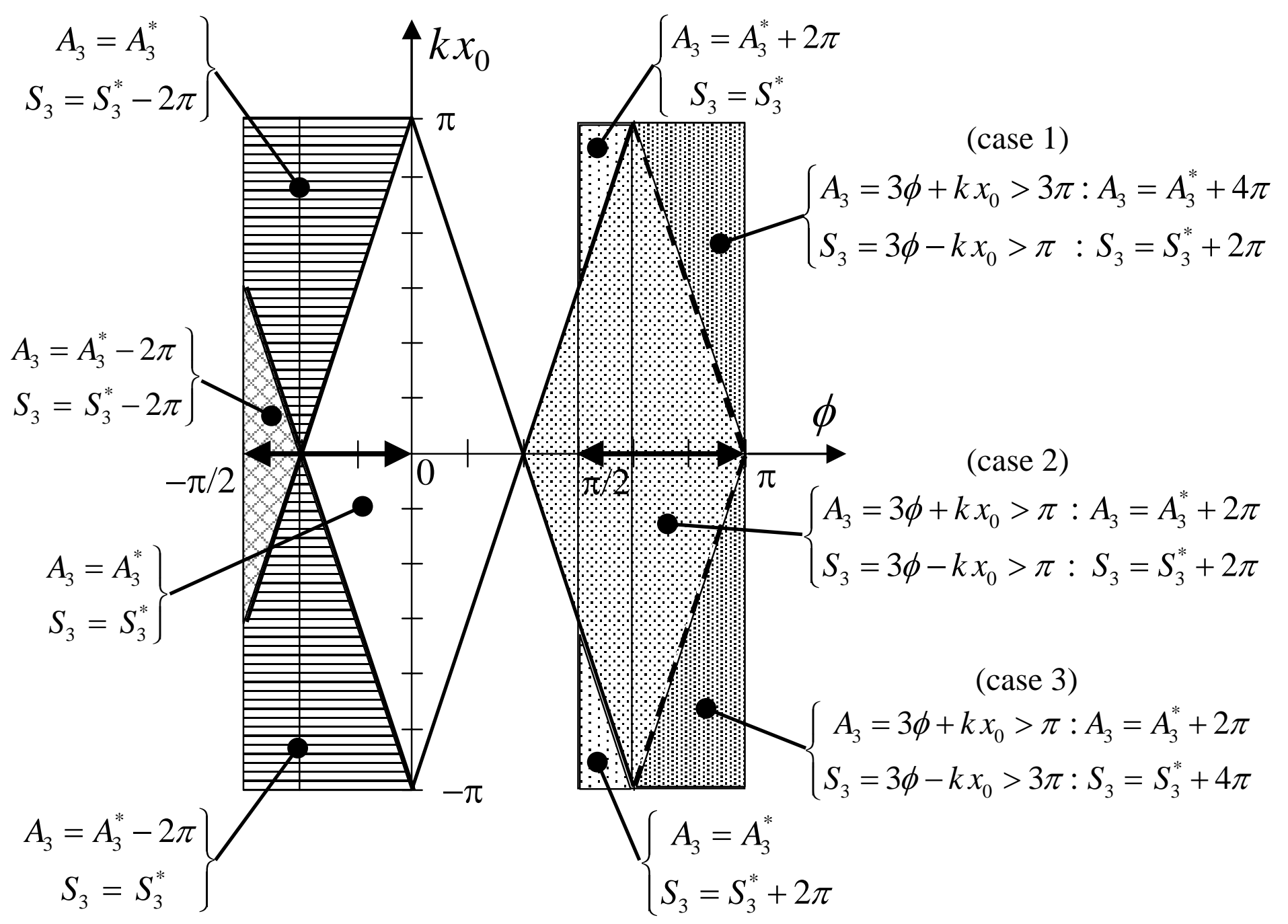

Fig. A1 Map of angle corrections required for $A_{3}{ }^{*}$ and $S_{3}{ }^{*}$ when $\phi$ and $k x_{0}$ are given 


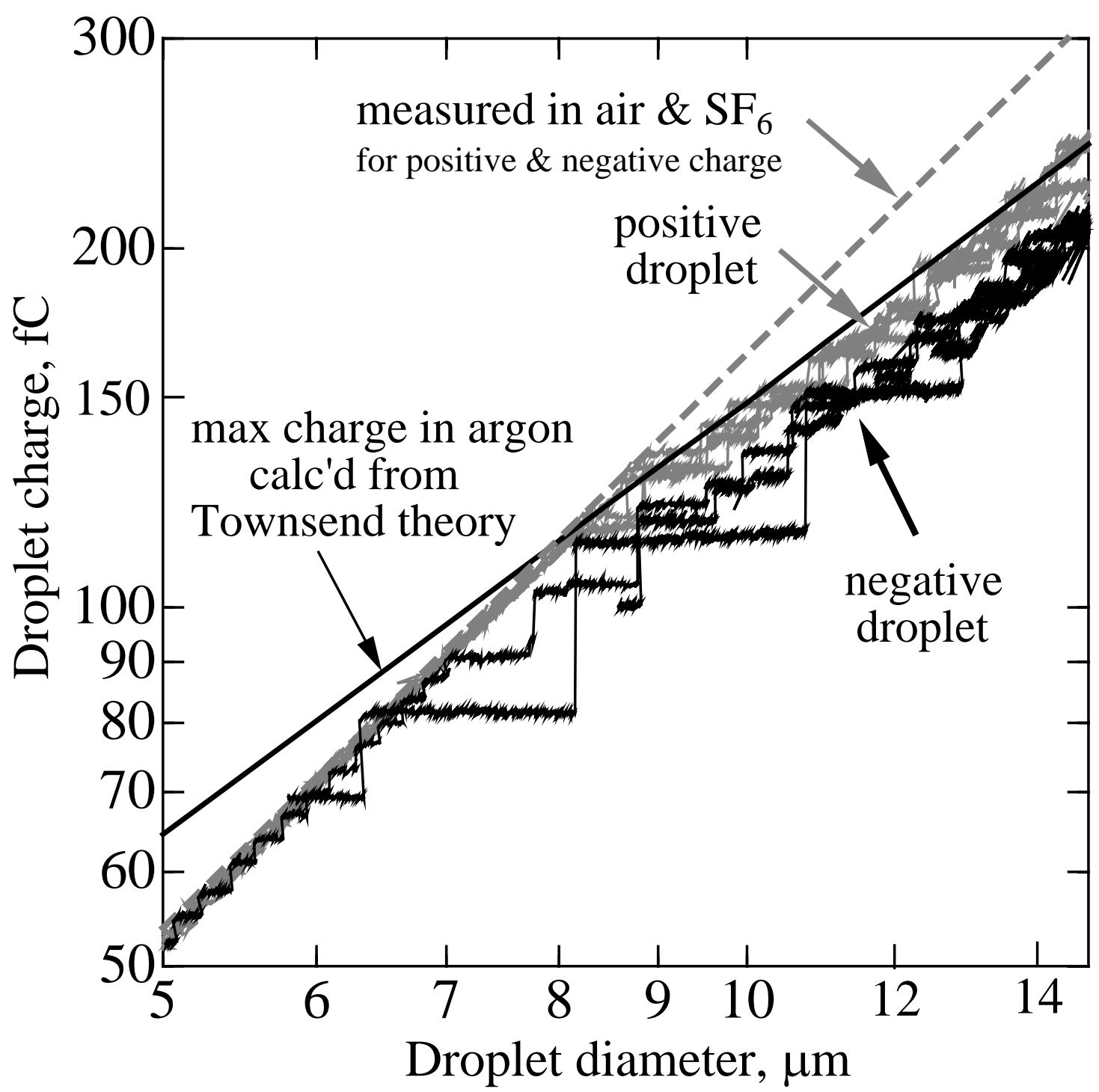

Fig. B1 Electric breakdown observed in Argon gas 